\title{
Diffusion Rates in Inorganic Nuclear Materials
}

\author{
A. L. Dragoo \\ Institute for Materials Research, National Bureau of Standards, Washington, D.C. 20234
}

(December 13, 1967)

\begin{abstract}
The tracer diffusion coefficient, the self-diffusion coefficient, the intrinsic diffusion coefficient and the interdiffusion coefficient are briefly described. Grain boundary and lattice (volume) diffusion are contrasted. The frequency factors $\left(D_{0}\right)$ and activation energies $(Q)$ are tabulated for diffusion in the borides, carbides, and oxides of $\mathrm{Be}, \mathrm{Hf}, \mathrm{Mo}, \mathrm{Nb}, \mathrm{Ta}, \mathrm{Th}, \mathrm{Ti}$, and $\mathrm{Zr}$ and for diffusion of $\mathrm{C}, \mathrm{N}$, and $\mathrm{O}$ in these metals. The purity of the solvent media, the preparation and properties of the samples, the method, the type of diffusion coefficient measured and the temperature range are also specified.
\end{abstract}

Key Words: Chemical interdiffusion, grain-boundary diffusion, intrinsic diffusion, lattice diffusion, self-diffusion, tracer diffusion.

\section{Introduction}

Since diffusion is often the rate controlling step in kinetic processes in solids-such as sintering, creep, precipitation, and corrosion-diffusion data have a necessary part in deciding the role of many metals and their compounds in high-temperature nuclear technology. Borides, carbides, nitrides, and oxides of plutonium and uranium, as well as the alloys of $\mathrm{Pu}$ and $\mathrm{U}$ with refractory metals, are potential high-temperature nuclear fuels. Refractory metals, such as Hf, Mo, $\mathrm{Nb}, \mathrm{Ta}, \mathrm{Ti}$, and $\mathrm{Zr}$ also may be used as structural and cladding materials. Thorium compounds can be transmuted to ${ }^{233} \mathrm{U}$-fuels in breeder reactors, and $\mathrm{BeO}$ can serve as a neutron moderator and reflector.

In this compilation diffusion data are presented for the borides, carbides, and oxides of Be, $\mathrm{Hf}, \mathrm{Mo}, \mathrm{Nb}$, Ta, Th, Ti, and Zr. No diffusion data are available to date for the nitrides nor for plutonium compounds. A similar compilation of the very extensive data for uranium compounds is now in progress. Since corrosion of the metals by $\mathrm{C}, \mathrm{N}$, and $\mathrm{O}$ often proceeds by reactive diffusion, diffusion data for $\mathrm{C}, \mathrm{N}$, and $\mathrm{O}$ diffusion in the eight metals listed above have been included (data for B diffusion in these metals were not available).

\section{Diffusion Coefficients}

In general, a diffusion coefficient $D$ is defined by Fick's first law as the constant which relates the flux of matter $J$ to the concentration gradient causing the flow. The gradient usually is taken to be only in the $x$ direction, thus

$$
J=-D(\partial C / \partial x)
$$

In the case of solids, three different diffusion coefficients - tracer diffusion $\left(D_{T}\right)$, intrinsic diffusion $\left(D_{i}\right)$ and chemical interdiffusion $(\tilde{D})$ - can be considered depending on the experimental conditions. The tracer and intrinsic diffusion coefficients apply to the diffusion of a single species; whereas the interdiffusion coefficient applies to the intermingling of species.

In the case of tracer diffusion, only a trace amount of species " $i$ " diffuses into a homogeneous solid from a very thin layer at the surface of the sample. In the absence of any external force field and of the influence of other species, the $i$-atoms will diffuse in a random manner toward an equilibrium distribution due only to their own concentration gradient $\left(\partial C_{i} / \partial x\right)$. If the tracer is the same as one of the elements of the solid matrix, $D_{T}$ becomes the self-diffusion coefficient, $D^{*}$. The tracer and self-diffusion coefficients can be determined by a wide variety of techniques which are noted in the tables.

The intrinsic diffusion coefficient takes into account the atom flux resulting from (1) the redistribution described by $D_{T},(2)$ the effect of a nonideal and nonhomogeneous solid solution of " $i$," and (3) the influence of the driving forces of the other species on the diffusion of " $i$." When substituted into eq $(1), D_{i}$ describes the flux of " $i$ " with respect to a local lattice plane in a nonhomogeneous, nonideal, solid solution which is not subject to any external driving force. The driving forces in a nonideal, nonhomogeneous solid solution are the chemical potential gradients $\left(\partial \mu_{i} / \partial x\right)$ of the various constituents of the material. The intrinsic diffusion coefficient generally is not measured experimentally, but it can be calculated if tracer diffusion coefficients of the species and the variation of the activity coefficient $\gamma_{i}$ with respect to the mole fraction $N_{i}$ of " $i$ " are known. 
If the rates of diffusion of different ionic species are not equalized by an internal potential gradient or by external forces, their interdiffusion will be accompanied by a net flow of imperfections. This flow of imperfections can be observed by inert marker techniques as a net movement of the lattice with respect to some fixed plane (e.g., the end of the sample). Thus, if the net flow of species is measured with respect to a fixed plane outside the diffusion zone, diffusion and the movement of the local lattice planes together will be measured. The chemical interdiffusion coefficient (or merely the "chemical diffusion coefficient") $\widetilde{D}$ is obtained from eq (1) if the flux $J$ in this equation is measured with respect to a fixed plane outside the diffusion zone. If it is assumed that $\widetilde{D}$ is a function of the ratio $x / \sqrt{t}$, where $t$ is time, $\widetilde{D}$ can be determined by the classical method of Boltzmann $[1]^{1}$ and Matano [2]. If there is no net flow of imperfections during diffusion, $\widetilde{D}$ for interdiffusion in a binary system equals the intrinsic diffusion coefficients of the two species $\left(D_{12}\right.$ will be used in this case).

It should be emphasized that $D_{T}$ can be determined only in a homogeneous solid. If the solid is nonhomogeneous, then $\tilde{D}$, and in some cases $D_{i}$, can be determined if the flux with respect to a fixed plane is known. These three diffusion coefficients apply to diffusion through the lattice.

In addition, diffusion can take place along grain boundaries. Grain-boundary diffusion of a tracer into a solid gives a nearly linear [3-6] decrease of $\ln C_{i}$ with penetration depth $x$, whereas $\ln C_{i}$ varies linearly with $x^{2}$ for lattice (volume) diffusion. The grainboundary diffusion coefficient is represented in the tables by $D_{\mathrm{gr}}$.

Diffusion coefficients for the borides, carbides, and oxides of Be, Ti, Zr, Hf, Nb, Ta, Mo, and Th have been measured generally for the cations and occasionally for the anions. The self-diffusion and tracer diffusion coefficients correspond empirically to the Arrhenius relation

$$
D=D_{0} \exp (-Q / R T)
$$

where $D_{0}$ is the frequency factor, $Q$ is the activation energy, $R$ is the ideal gas constant and $T$ is the absolute temperature. Although the chemical and intrinsic diffusion coefficients may be expressed in terms of eq $(2), \tilde{D}$ and $D_{i}$ have a more complex temperature dependence.

\section{Experimental Methods}

In general, there are two modes of determining $D$. The direct method measures the distribution of concentration through the solid at a time $t$. The indirect method determines the diffusion coefficient by measuring a diffusion controlled phenomenon: attenuation of radioactivity, exchange of isotopes between a solid

${ }^{1}$ Figures in brackets indicate the literature references at the end of this paper and a gas, release of anelastic strain, change of a physical property of the surface or the release of rare gases.

\section{Reliability}

Most of the diffusion values in the tables are "preliminary." That is, they are the only values presently available, and they will suffice for rough estimates of the behavior of materials in technological applications.

The immediate needs of technology have provided the rationale for including many of the diffusion values in this compilation. Since the ratios of two independent measurements of the diffusion coefficient can be as great as $10^{6}$ (see $\mathrm{Be}$ diffusion in $\mathrm{BeO}$, low temperatures) due primarily to differences in materials and experimental methods, caution is required in the use of diffusion values. To this end, diffusion values from more than one source have been tabulated when they have been available and auxiliary information and comments have been included in the tables.

To go beyond the rough estimate to the fundamental understanding of the properties of solids requires that a very exacting set of criteria be placed upon materials and methods. The solvent medium should be a very pure, well-characterized single crystal. In the case of chemical diffusion, the concentration dependence of the diffusion coefficient should be determined or the diffusion coefficient should be determined at given concentrations. Indirect determinations of $D$ should be compared with direct determinations of $D$ from chemical or isotopic concentration profiles. Measurements of a physical property related to the concentration do not establish the concentration profile unless the property's functional dependence on concentration is determined (e.g., the assumption of linearity is not sufficient). Diffusion anneals should be carried out above one-half the melting point temperature so that extrinsic diffusion is avoided, unless impurity effects are to be measured. These anneals must be carried out under thermodynamically specifiable conditions - temperatures and partial pressures must be known.

The scope of this compilation prevented a detailed use of these criteria in establishing the degree of reliability of each diffusion coefficient. Critical evaluations also were limited by the lack of first-hand experience with most of the experimental techniques, and by the absence of experimental details, of tabulated data and of careful error analyses in many of the original works. Nevertheless, some general comments can be made.

Much of the unreliability results from the specimens used, and high impurity concentrations are foremost among the causes. Impurity controlled diffusion rates (extrinsic diffusion) are to be expected when impurities with valences other than that of the diffusing species are present in concentrations greater than $100 \mathrm{ppm}$ and when the annealing temperature is low with respect to the melting point. Diffusion coefficients measured under such conditions are strictly only characteristic of the specimen studied and are not "intrinsic" to the material. 
Several errors can arise from the use of polycrystalline materials. Grain boundaries and pores provide high diffusivity paths. Experimental observations of these effects and experiments in which these may have been a major factor have been noted in the tables. These high diffusivity paths probably contributed measureably to the observed rates in experiments carried out below 700 to $1000{ }^{\circ} \mathrm{C}$, but it has not been possible to assess the magnitude of these contributions here. Grain orientation is important when the material has less than cubic symmetry because the diffusion coefficient is a second rank tensor and, therefore, depends on crystallographic direction.

Dislocations, subgrain boundaries and microcleavages due to surface damage are other sources of high diffusivity paths which can occur in both single and polycrystalline specimens. These sources of error have been overlooked by most investigators.

Diffusion coefficients from any experiments in which chemical concentration gradients were present, such as oxidation of metals, have been classed as chemical diffusion coefficients. Apparent agreement between such diffusion coefficients and self-diffusion coefficients should be considered fortuitous. Furthermore, when these diffusion coefficients have been obtained by assuming that they are independent of concentration, they are first order approximations for the overall diffusion zone in the specimen studied.

Experimental methods are the second area in which problems arise. Indirect measurements usually depend upon the change of a physical property which is assumed to depend on concentration. In some cases, notably anelastic strain measurements, such methods can be very reliable. However, indirect methods usually do not reveal the concentration dependence of the chemical diffusion coefficient nor the presence of high diffusivity paths.

In regard to both indirect and direct methods, the functional dependence of the measured property on concentration often has not been demonstrated and the influence of other factors has not been determined. Microhardness, for example, depends on microstructure, impurities, and preferred orientation of the grains as well as on chemical composition, and it is not necessarily a linear function of concentration. The absorption of beta radiation by a solid is generally more complex than a mere exponential function of distance. If the functional relationship only has been assumed, the reported diffusion coefficient should be considered a preliminary value.
Some other sources of unreliability which the user of the tables should determine from the original work if accuracy is very important are:

(1) temperature control and measurement;

(2) failure to satisfy the boundary conditions of the diffusion problem;

(3) change in the characteristics of the specimen during the diffusion anneal due to sintering, vaporization, annihilation or production of defects and reaction with components of the furnace; and

(4) hysteresis in the response of the measuring instrument.

\section{Key to the Tables}

Each table contains data for a metal and its borides, carbides, and oxides. The data in each table is ordered first according to the solvent medium, i.e., the matrix metal or compound. First the matrix metal is presented, followed in turn by the borides, the carbides and the oxides. For example, in the table for thorium, thorium metal occurs first followed by $\mathrm{ThO}_{2}$ (there is no data for the borides and carbides).

The data for the diffusion in the metallic solvent medium is ordered alphabetically with respect to the diffusing element (C, N, or O). A further ordering with respect to the metallic phase is used if this distinction is important (e.g., C-diffusion in $\alpha$ - $\mathrm{Ti}$ precedes Cdiffusion in $\beta$-Ti).

The compound solvent medium (e.g., oxides) is subdivided first with respect to the number of cations per molecule. For example, the niobium carbides have the following order: $\mathrm{NbC} \rightarrow \mathrm{Nb}_{2} \mathrm{C} \rightarrow \mathrm{Nb}_{4} \mathrm{C}_{3} \rightarrow$ mixed carbides. Each compound subdivision next is arranged alphabetically with respect to the diffusing element. The order for $\mathrm{BeO}$ is: $\mathrm{Be} \rightarrow \mathrm{O} \rightarrow \mathrm{T}\left({ }^{3} \mathrm{H}\right) \rightarrow$ Xe.

In addition to specifying the solvent medium and diffusing element, each entry gives the purity of the solvent medium, the preparation and properties of the diffusion sample, the method used to measure the diffusion coefficient, the type of diffusion coefficient measured, the temperature range, the frequency factor $\left(D_{0}\right)$ and the activation energy $(Q)$, in $\mathrm{cal} / \mathrm{mol}$ $(1$ cal $=4.184 \mathrm{~J})$.

I thank J. R. Manning for the many helpful discussions during the preparation of this compilation. This work was partially supported by the Advanced Research Projects Agency, U.S. Department of Defense. 
Beryllium

\begin{tabular}{|c|c|c|c|c|c|c|c|c|c|c|}
\hline $\begin{array}{l}\text { Solvent } \\
\text { medium }\end{array}$ & $\begin{array}{c}\text { Diffusing } \\
\text { element }\end{array}$ & Purity ${ }^{a}$ & $\begin{array}{l}\text { Preparation and } \\
\text { properties }\end{array}$ & Method & $\begin{array}{l}\text { Diffusion } \\
\text { coefficient }\end{array}$ & $\begin{array}{l}\text { Temperature } \\
\text { range, }{ }^{\circ} \mathrm{C}\end{array}$ & $D_{0} \mathrm{~cm}^{2} / \mathrm{s}$ & $Q \mathrm{cal} / \mathrm{mol}$ & Comments & Ref. \\
\hline $\mathrm{BeO} \ldots \ldots$ & $\mathrm{Be}^{\mathrm{b}} .$. & $\begin{array}{l}\mathrm{Al}, 15 ; \mathrm{Fe}, 200 \\
\mathrm{~Pb}, 20 ; \mathrm{Si}, 500\end{array}$ & $\begin{array}{l}\text { Polycrystalline, } \\
\text { cold-pressed and } \\
\text { sintered, 96-98\% } \\
\text { of theoretical } \\
\text { density. }\end{array}$ & $\begin{array}{l}\text { Determination of } \\
\text { the radioisotope } \\
\text { distribution by } \\
\text { sectioning. }\end{array}$ & $\begin{array}{l}D^{*} \text { (self- } \\
\text { diffusion). }\end{array}$ & $1180-1800$ & $2.49 \times 10^{-3}$ & 62,500 & $\begin{array}{l}\text { Grain-boundary } \\
\text { diffusion was } \\
\text { considered insig- } \\
\text { nificant because } \\
\text { grain size } \\
\text { exceeded diffusion } \\
\text { depth; impurity } \\
\text { content was prob- } \\
\text { ably important. }\end{array}$ & [1] \\
\hline $\mathrm{BeO} \ldots$ & Be.......... & $\begin{array}{l}\mathrm{Al}, 3000 ; \mathrm{Ca} \\
\quad 330 ; \mathrm{Fe}<25 \\
\mathrm{Si}<77 ; \mathrm{Ti}, 61 \\
\mathrm{P}<500\end{array}$ & $\begin{array}{l}\text { Polycrystalline, } \\
\text { slip cast and } \\
\text { sintered } 92-93 \% \text { of } \\
\text { theoretical density. }\end{array}$ & $\begin{array}{l}\text { Determination of } \\
\text { the radioisotope } \\
\text { distribution by } \\
\text { sectioning. }\end{array}$ & $\begin{array}{l}D^{*} \\
D^{*}\end{array}$ & $\begin{array}{l}1550-1730 \\
1730-1960\end{array}$ & $\begin{array}{l}5.56 \times 10^{-3} \\
6.14 \times 10^{-2}\end{array}$ & $\begin{array}{r}111,600 \\
66,100\end{array}$ & $\begin{array}{l}\text { Variation in } \\
\text { diffusion coefficient } \\
\text { was primarily due } \\
\text { to impurity con- } \\
\text { tent; scatter in } \\
\text { data makes the }\end{array}$ & [2] \\
\hline $\mathrm{BeO} \ldots \ldots$ & Вe.......... & $\begin{array}{l}\text { Al }<160 ; \mathrm{Fe}, 43 \\
\quad \mathrm{Si}, 60 ; \mathrm{Ti}, 33 \\
\text { P }<500\end{array}$ & $\begin{array}{l}\text { Polycrystalline, } \\
\text { hot-pressed, } 99.3 \% \\
\text { of theoretical } \\
\text { density. }\end{array}$ & $\begin{array}{l}\text { Determination of } \\
\text { the radioisotope } \\
\text { distribution by } \\
\text { sectioning. }\end{array}$ & $\begin{array}{l}D^{*} \\
D^{*}\end{array}$ & $\begin{array}{l}1550-1725 \\
1725-2000\end{array}$ & $\begin{array}{l}1.35 \\
1.07 \times 10^{-6}\end{array}$ & $\begin{array}{l}92,000 \\
36,000\end{array}$ & $\begin{array}{l}\text { actual magnitude of } \\
\text { curvature uncer- } \\
\text { tain. "Tailing"" } \\
\text { of concentration }\end{array}$ & [2] \\
\hline $\mathrm{BeO} \ldots$ & $\mathrm{Be} \ldots$ & $\begin{array}{l}\text { Mo micro- } \\
\text { inclusions; all } \\
\text { other impurities } \\
\text { under } 100 .\end{array}$ & $\begin{array}{l}\text { Single crystal; } \\
\text { grown in lithium } \\
\text { molybdate flux. }\end{array}$ & $\begin{array}{l}\text { Determination of } \\
\text { the radioisotope } \\
\text { distribution by } \\
\text { sectioning. }\end{array}$ & $\begin{array}{l}D^{*} \\
D^{*}\end{array}$ & $\begin{array}{l}1500-1760 \\
1720-1975\end{array}$ & $\begin{array}{l}1.27 \times 10^{-3} \\
1.23 \times 10^{-6}\end{array}$ & $\begin{array}{l}64,000 \\
36,000\end{array}$ & $\begin{array}{l}\text { distribution } \\
\text { curve in single } \\
\text { crystal specimens } \\
\text { (which is sugges- } \\
\text { tive of grain } \\
\text { boundary diffusion } \\
\text { in polycrystalline } \\
\text { samples) was } \\
\text { ascribed to micro- } \\
\text { cleavages due to } \\
\text { grinding. }\end{array}$ & {$[2,3]$} \\
\hline $\mathrm{BeO} \ldots \ldots \ldots$ & $0 \ldots \ldots \ldots$ & $\begin{array}{l}\mathrm{Al}, 3000 ; \mathrm{Ca}, 330 ; \\
\quad \mathrm{Fe}<25 ; \mathrm{Si} \\
<77 ; \mathrm{Ti}, 6 \mathrm{l} \\
\mathrm{P}<500\end{array}$ & $\begin{array}{l}\text { Polycrystalline, } \\
\text { slip cast and } \\
\text { sintered, 92-93\% } \\
\text { of theoretical } \\
\text { density. }\end{array}$ & $\begin{array}{l}\text { Heterogeneous } \\
\text { isotopic exchange } \\
\text { between a solid } \\
\text { sample and a gas. }\end{array}$ & $D^{*}$ & $1600-1900$ & $5.2 \times 10^{-7}$ & 42,900 & $\begin{array}{l}\text { Evidence of signifi- } \\
\text { cant grain- } \\
\text { boundary diffusion; } \\
\text { the rates may also } \\
\text { be affected by the } \\
\text { impurity concen- } \\
\text { tration. }\end{array}$ & {$[2,4,5]$} \\
\hline $\mathrm{BeO} \ldots \ldots$ & $\mathrm{O} \ldots \ldots \ldots$ & $\begin{array}{l}\mathrm{Fe}, 25 ; \mathrm{Li}, 20 ; \\
\quad \text { Mo, } 1200 ; \text { all } \\
\text { others } \leqq 10\end{array}$ & $\begin{array}{l}\text { Single crystal; } \\
\text { grown in a } \\
\text { molybdate flux. }\end{array}$ & $\begin{array}{l}\text { Heterogeneous } \\
\text { isotopic exchange } \\
\text { between a solid } \\
\text { sample and a gas. }\end{array}$ & $D^{*}$ & $1300-1700$ & $2.95 \times 10^{-5}$ & 68,500 & $\begin{array}{l}\text { Grain boundary } \\
\text { effects were } \\
\text { removed by use } \\
\text { of single crystal. }\end{array}$ & [6] \\
\hline $\mathrm{BeO} \ldots \ldots \ldots$ & $\mathrm{T}\left({ }^{3} \mathrm{H}\right) \ldots$ & Not stated. & $\begin{array}{l}\text { Commerical BeO } \\
\text { powder } \\
\text { (Brush UOX). }\end{array}$ & $\begin{array}{l}\text { Rate of fission } \\
\text { gas release from } \\
\text { irradiated } \\
\text { powder, spheri- } \\
\text { cal particles } \\
\text { were assumed. }\end{array}$ & $\begin{array}{c}D_{T} \text { (tracer } \\
\quad \text { diffusion) }\end{array}$ & $673-923$ & $6.6 \times 10^{-13}$ & 24,500 & $\begin{array}{l}\mathrm{T} \text { was assayed as } \\
\mathrm{T}_{2} \mathrm{O} \text {, recovery as } \\
\mathrm{T}_{2} \mathrm{O} \text { was quantita- } \\
\text { tive. The very } \\
\text { low values for } \\
\text { both } D_{0} \text { and } Q \text { are } \\
\text { suggestive of sig- } \\
\text { nificant grain } \\
\text { boundary diffusion. }\end{array}$ & [7] \\
\hline $\mathrm{BeO} \ldots$ & T............. & Not stated. & $\begin{array}{l}\text { Commerical BeO } \\
\text { powder (Brush } \\
\text { Minox). }\end{array}$ & $\begin{array}{l}\text { Rate of fission } \\
\text { gas release from } \\
\text { irradiated } \\
\text { powder, spheri- } \\
\text { cal particles } \\
\text { were assumed. }\end{array}$ & $D_{T}$ & $673-873$ & $7.6 \times 10^{-13}$ & 24,000 & & [7] \\
\hline $\mathrm{BeO} \ldots \ldots$ & $\mathrm{T} \ldots \ldots \ldots$ & Not stated. & $\begin{array}{l}\text { Sintered compacts } \\
\text { and powders were } \\
\text { investigated; } \\
\text { powder data was } \\
\text { considered more } \\
\text { reliable. }\end{array}$ & $\begin{array}{l}\text { Rate of fission } \\
\text { gas release from } \\
\text { irradiated } \\
\text { powder, spheri- } \\
\text { cal particles } \\
\text { were assumed. }\end{array}$ & $D_{T}$ & $700-1000$ & 0.131 & 70,000 & $\begin{array}{l}\text { Data indicated a } \\
\text { lower activation } \\
\text { energy below } 700 \\
{ }^{\circ} \mathrm{C} ; \mathrm{T} \text { was assayed } \\
\text { as HT, a correc- } \\
\text { tion was required } \\
\text { for the loss as } \\
\text { HTO. }\end{array}$ & [8] \\
\hline $\mathrm{BeO}$.. & $\mathrm{Xe} \ldots \ldots$ & $\begin{array}{l}\text { Samples were } \\
\text { obtained from } \\
\text { S. B. Austerman, } \\
\text { Ref. } 2,3 \text {. }\end{array}$ & $\begin{array}{l}\text { Pyramidal, single- } \\
\text { crystals, were } \\
\text { assumed to be } \\
\text { spherical for } \\
\text { calculation of } D .\end{array}$ & \begin{tabular}{|l|} 
Hahn emanation \\
technique, Xe \\
recoiled into \\
oxide when oxide \\
was irradiated \\
in contact with \\
$\mathrm{UO}_{2}$.
\end{tabular} & $D_{T}$ & $800-1500$ & $3.0 \times 10^{-6}$ & 63,500 & & [9] \\
\hline
\end{tabular}

a Impurity concentrations are given in ppm unless otherwise stated.

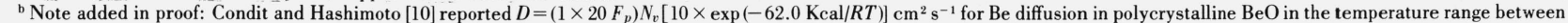
1330 to $2135^{\circ} \mathrm{C} . F_{p}$ is the fraction of porosity and $N_{v}$ is the molar fraction of vacancies introduced by impurities.

[1] H. J. DeBruin and G. M. Watson, J. Nucl. Mater. 14, 239 (1964); U.S. At. Energy Comm. Report ORNL-3526 (1964).

[2] S. B. Austerman, J. Nucl. Mater. 14, 248 (1964); U.S. At. Energy Comm. Report NAA-SR-5893 (1961)

[2] S. B. Austerman, J. Nucl. Mater. 14, 248 (1964); U.S. At. Energy Comm
[3] S. B. Austerman and J. W. Wagner, J. Am. Ceram. Soc. 49, 94 (1966).

[4] S. B. Austerman and J. W. Wagner, J. Am. Ceram. Soc. 49, 94 (1966).

[5] S. B. Austerman and R. A. Meyer, U.S. At. Energy Comm. Report NAA-SR-7637 (1962)

[6] J. B. Holt, J. Nucl. Mater. 11,107 (1964).

[7] A. R. Palmer, D. Roman, and H. J. Whitfield, J. Nucl. Mater. 14, 141 (1964).

[8] E. Rothwell and L. L. Wassell, J. Nucl. Mater. 16, 208 (1965).

[9] D. L. Morrison, T. S. Elleman, and D. N. Sunderman, J. Appl. Phys. 35, 1616 (1964).

[10] R. H. Condit and Y. Hashimuto, J. Am. Ceram. Soc. 50, 425 (1967). 


\section{Hafnium}

\begin{tabular}{|c|c|c|c|c|c|c|c|c|c|c|}
\hline $\begin{array}{l}\text { Solvent } \\
\text { medium }\end{array}$ & $\begin{array}{l}\text { Diffusing } \\
\text { element }\end{array}$ & Purity ${ }^{a}$ & $\begin{array}{l}\text { Preparation and } \\
\text { properties }\end{array}$ & Method & $\begin{array}{c}\text { Diffusion } \\
\text { coefficient }\end{array}$ & $\begin{array}{l}\text { Temperature } \\
\text { range, }{ }^{\circ} \mathrm{C}\end{array}$ & $D_{0} \mathrm{~cm}^{2} / \mathrm{s}$ & $Q \mathrm{cal} / \mathrm{mol}$ & Comments & Ref. \\
\hline $\mathrm{Hf}_{. . \ldots \ldots \ldots}$ & o..... & $\begin{array}{l}\mathrm{Zr}<100 ; \mathrm{O} \\
\quad 120 ; \mathrm{N}, 16 ; \mathrm{C} \\
\quad 37\end{array}$ & $\begin{array}{l}\text { Zone-refined; oxide } \\
\text { layer formed by } \\
\text { anodization. }\end{array}$ & $\begin{array}{l}\text { Time rate of } \\
\text { change of inter- } \\
\text { ference color } \\
\text { (i.e., thickness) } \\
\text { of oxide film on } \\
\text { metal due to } \\
\text { solution of film } \\
\text { in metal. }\end{array}$ & $\begin{array}{l}\widetilde{D} \text { (chemical } \\
\text { diffusion). }\end{array}$ & $500-1050$ & 0.660 & 50,820 & $\begin{array}{l}\tilde{D} \text { assumed to be } \\
\text { independent of } \\
\text { concentration. }\end{array}$ & [1] \\
\hline Hf............. & $\mathrm{O} \ldots \ldots \ldots$ & $\begin{array}{l}\mathrm{Zr}, 1.0 \text { w/o; } \mathrm{Al}, \\
\mathrm{1100} ; \mathrm{Fe}, 450 ; \\
\mathrm{Cu}, 60 ; \mathrm{Cr}, 800 ; \\
\mathrm{Mg}, 50 ; \mathrm{Ti}, 300 ; \\
\text { Ni, 60; all } \\
\text { others < } 50 .\end{array}$ & $\begin{array}{l}\text { Hot-rolled and } \\
\text { annealed at } 800^{\circ} \mathrm{C} ; \\
\text { machined and } \\
\text { polished; } \\
\text { oxidized in air. }\end{array}$ & $\begin{array}{l}\text { Determination of } \\
\text { concentration } \\
\text { gradient by micro- } \\
\text { hardness measure- } \\
\text { ments. }\end{array}$ & $D$ & $700-1200$ & 1.6 & 49,300 & $\begin{array}{l}\text { Assumption of } \\
\text { linear relation- } \\
\text { ship between } \\
\text { oxygen concentra- } \\
\text { tion and hardness } \\
\text { probably was not } \\
\text { justified, according } \\
\text { to [4] the } \\
\text { relationship is } \\
\text { parabolic; nitrogen } \\
\text { gradient was super- } \\
\text { imposed on oxygen } \\
\text { gradient. }\end{array}$ & [2] \\
\hline $\begin{array}{l}\mathrm{Hf}_{0.86}-\ldots . \\
\mathrm{Ca}_{0.14}-\ldots . \\
\mathrm{O}_{1.86}\end{array}$ & $\begin{array}{c}0 \ldots \ldots \\
\ldots \ldots \ldots \ldots \\
\ldots \ldots\end{array}$ & $\begin{array}{l}\mathrm{Fe}_{2} \mathrm{O}_{3}, 0.012- \\
0.1 \% ; \mathrm{Al}_{2} \mathrm{O}_{3}, \\
0.012-0.1 \% \\
\mathrm{TiO}_{2}, 0.01-0.2 \% \\
\mathrm{ZrO}_{2}, 2.5-3 \% ; \\
\mathrm{Nb}_{2} \mathrm{O}_{5}, 0.05-0.1 \% \\
\text { (weight percent). }\end{array}$ & $\begin{array}{l}\text { Tube closed at } \\
\text { one end; density } \\
9.51 \mathrm{~g} / \mathrm{cm}^{3} ; \\
\text { porosity } \sim 5 \% \text {. }\end{array}$ & $\begin{array}{l}\text { Determination of } \\
\text { the rate of } \\
\text { permeation of } \\
\mathrm{O}_{2} \text { through walls } \\
\text { of stabilized } \\
\text { hafnia tube. }\end{array}$ & $\tilde{D}$ & $1100-1600$ & 22 & 55,700 & $\begin{array}{l}\text { A large drop in } \\
\text { partial pressure } \\
\text { between the inside } \\
\text { and outside of the } \\
\text { tube caused a large } \\
\text { chemical potential } \\
\text { gradient. }\end{array}$ & {$[3]$} \\
\hline
\end{tabular}

${ }^{a}$ Impurity concentrations are given in ppm unless otherwise stated.

[1] J. P. Pemsler, J. Electrochem. Soc. 111, 1185 (1964); J. Electrochem. Soc. 106, 1067 (1959).

[2] J. D. Gadd and E. B. Evans, Corrosion 17, 441t (1961),

[3] A. W. Smith. F. W. Meszaros, and C. D. Amata, J. Am. Ceram. Soc. 49, 240 (1966).

[4] E. Rudy and P. Stecher, J. Less-Common Metals 5, 78 (1963).

\section{Molybdenum}

\begin{tabular}{|c|c|c|c|c|c|c|c|c|c|c|}
\hline $\begin{array}{l}\text { Solvent } \\
\text { medium }\end{array}$ & $\begin{array}{c}\text { Diffusing } \\
\text { element }\end{array}$ & Purity ${ }^{a}$ & $\begin{array}{l}\text { Preparation and } \\
\text { properties }\end{array}$ & Method & $\begin{array}{c}\text { Diffusion } \\
\text { coefficient }\end{array}$ & $\begin{array}{c}\text { Temperature } \\
\text { range, }{ }^{\circ} \mathrm{C}\end{array}$ & $D_{0} \mathrm{~cm}^{2} / \mathrm{s}$ & $Q \mathrm{cal} / \mathrm{mol}$ & Comments & Ref. \\
\hline $\mathrm{Mo}_{2} \mathrm{~B} \ldots \ldots \ldots$ & B....... & Not stated. & $\begin{array}{l}\text { Boride layer was } \\
\text { formed on a Mo } \\
\text { cylinder by boro- } \\
\text { nizing in boron } \\
\text { carbide and borax. }\end{array}$ & $\begin{array}{l}\text { Determination of } \\
\text { the concentration } \\
\text { gradient by } \\
\text { microhardness } \\
\text { measurements. }\end{array}$ & $\tilde{D} \begin{array}{c}\text { (chemical } \\
\text { diffusion) }\end{array}$ & $1100-1400$ & 0.0696 & 45,000 & $\begin{array}{l}\tilde{D} \text { was assumed to } \\
\text { be independent of } \\
\text { concentration; } \\
\text { absence of a homo- } \\
\text { geneity range [2] was } \\
\text { probably a major } \\
\text { source of error. }\end{array}$ & [1] \\
\hline $\mathrm{Mo}_{2} \mathrm{C} \ldots \ldots$ & C... & Not stated. & $\begin{array}{l}\text { Carbide layer was } \\
\text { formed on a Mo } \\
\text { cylinder by car- } \\
\text { burizing in } \\
\text { graphite. }\end{array}$ & $\begin{array}{l}\text { Determination of } \\
\text { the concentra- } \\
\text { tion gradient by } \\
\text { microhardness } \\
\text { measurements. }\end{array}$ & $\tilde{D}$ & $1400-1700$ & 1,640 & 83,000 & $\begin{array}{l}\vec{D} \text { was assumed to } \\
\text { be independent of } \\
\text { concentration; } \\
\text { narrow homoge- } \\
\text { neity range [3]. }\end{array}$ & [1] \\
\hline
\end{tabular}

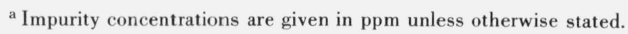

[1] G. V. Samsonov and A. P. Epik, Dopovidi Akad. Nauk Ukr. RSR [1], 67 (1964).

[2] M. Hansen and K. Anderko, Constitution of Binary Alloys, p. 253 (McGraw-Hill Book Co., New York, N.Y., 1958).

[3] ibid., p. 370.

Niobium

\begin{tabular}{|c|c|c|c|c|c|c|c|c|c|c|}
\hline $\begin{array}{l}\text { Solvent } \\
\text { medium }\end{array}$ & $\begin{array}{c}\text { Diffusing } \\
\text { element }\end{array}$ & Purity ${ }^{a}$ & $\begin{array}{l}\text { Preparation and } \\
\text { properties }\end{array}$ & Method & $\begin{array}{l}\text { Diffusion } \\
\text { coefficient }\end{array}$ & $\begin{array}{l}\text { Temperature } \\
\text { range, }{ }^{\circ} \mathrm{C}\end{array}$ & $D_{0} \mathrm{~cm}^{2} / \mathrm{s}$ & $Q \mathrm{cal} / \mathrm{mol}$ & Comments & Ref. \\
\hline $\mathrm{Nb} \ldots \ldots \ldots$ & $\mathrm{C} \ldots \ldots \ldots$ & Not stated. & Not stated. & $\begin{array}{l}\text { Measurement of } \\
\text { anelastic strain } \\
\text { by elastic after- } \\
\text { effect and by } \\
\text { internal friction. }\end{array}$ & $\begin{array}{c}D_{T} \text { (tracer } \\
\quad \text { diffusion) }\end{array}$ & $55-160$ & 0.015 & 27,000 & $\begin{array}{l}\text { Effects of } \\
\text { possible inter- } \\
\text { stitial impurity } \\
(\mathrm{C}, \mathrm{N}, \mathrm{O}) \text { inter- } \\
\text { actions were not } \\
\text { ascertained. }\end{array}$ & [1] \\
\hline $\mathrm{Nb} \ldots \ldots \ldots$ & $\mathrm{C} \ldots \ldots \ldots$ & $\begin{array}{l}\text { Nb, } 99.4 \text { w/o; C, } \\
1000(\max .) ; \mathrm{Ta}, \\
5000 ; \mathrm{Ti}, 400 ; \\
\mathrm{Si}, 200 ; \mathrm{Fe}, 100 . \\
\text { Degassed to } \\
\text { reduce } \mathrm{O} \text { and } \mathrm{N} \\
\text { damping peaks. }\end{array}$ & $\begin{array}{l}\text { Carbon introduced } \\
\text { by pyrolysis of } \\
\text { hydrocarbons, and } \\
\text { in a few samples } \\
\text { by heating wires } \\
\text { coated with graph- } \\
\text { ite-water slurry. }\end{array}$ & $\begin{array}{l}\text { Measurement of } \\
\text { anelastic strain } \\
\text { by elastic after- } \\
\text { effect and by } \\
\text { internal friction. }\end{array}$ & $D_{T}$ & $140-275$ & 0.004 & 33,020 & $\begin{array}{l}\text { Effects of } \\
\text { interestitial } \\
\text { impurity inter- } \\
\text { actions were } \\
\text { determined; } \\
\text { carbide precipi- } \\
\text { tation was } \\
\text { observed at "high" } \\
\text { C concentrations. }\end{array}$ & {$[2,3]$} \\
\hline
\end{tabular}

${ }^{a}$ Impurity concentrations are given in ppm unless otherwise stated. 
Niobium-Continued

\begin{tabular}{|c|c|c|c|c|c|c|c|c|c|c|}
\hline $\begin{array}{l}\text { Solvent } \\
\text { medium }\end{array}$ & $\begin{array}{l}\text { Diffusing } \\
\text { element }\end{array}$ & Purity ${ }^{a}$ & $\begin{array}{l}\text { Preparation and } \\
\text { properties }\end{array}$ & Method & $\begin{array}{c}\text { Diffusion } \\
\text { coefficient }\end{array}$ & $\begin{array}{c}\text { Temperature } \\
\text { range, },{ }^{\circ} \mathrm{C}\end{array}$ & $D_{0} \mathrm{~cm}^{2} / \mathrm{s}$ & $Q \mathrm{cal} / \mathrm{mol}$ & Comments & Ref. \\
\hline $\mathrm{Nb} . . .$. & C.. & $\begin{array}{l}\text { Nb, } 99.01 \text { w/o; } \\
\text { Ta, } 0.94 \text { w/o; O, } \\
400 ; \mathrm{Ti}, \mathrm{Fe}, \mathrm{Si} \\
\text { and } \mathrm{C} \text { not de- } \\
\text { tected. }\end{array}$ & $\begin{array}{l}\text { Porosity of metal } \\
\sim 13 \% ;{ }^{14} \mathrm{C} \text { labeled } \\
\text { emulsion pyrolyzed } \\
\text { on specimen, spec- } \\
\text { imens were cleaved } \\
\text { and were fastened } \\
\text { together in pairs } \\
\text { along active faces. }\end{array}$ & $\begin{array}{l}\text { Measurement of } \\
\text { attenuation of } \\
\beta \text {-radiation by } \\
\text { the method of } \\
\text { Gruzin [5] (ratio } \\
\text { of activities). }\end{array}$ & $D_{T}$ & $900-1100$ & $1.09 \times 10^{-5}$ & 32,000 & $\begin{array}{l}\text { High diffusivity } \\
\text { paths probably } \\
\text { existed along } \\
\text { pores. }\end{array}$ & [4] \\
\hline Nb....... & $\mathrm{N} \ldots .$. & $\begin{array}{l}\text { Nb, } 99.4 \text { w/o; C, } \\
1000(\max .) ; \mathrm{Ta}, \\
5000 ; \mathrm{Ti}, 400 ; \\
\mathrm{Si}, 200 ; \mathrm{Fe}, 100 . \\
\text { Degassed to } \\
\text { remove O and N } \\
\text { initially. }\end{array}$ & $\begin{array}{l}\mathrm{N} \text { added by absorp- } \\
\text { tion of molecular } \\
\text { nitrogen or } \mathrm{NH}_{3} \text {. }\end{array}$ & $\begin{array}{l}\text { Measurement of } \\
\text { anelastic strain } \\
\text { by elastic after- } \\
\text { effect and by } \\
\text { internal friction. }\end{array}$ & $D_{T}$ & $150-290$ & $8.6 \times 10^{-3}$ & 34,920 & $\begin{array}{l}\text { Effects of inter- } \\
\text { stitial impurity } \\
\text { interactions were } \\
\text { determined. }\end{array}$ & {$[2,3]$} \\
\hline $\mathrm{Nb} . .$. & $\mathrm{N} \ldots$ & Not stated. & $\begin{array}{l}\mathrm{N} \text { introduced by } \\
\text { heating a wire in } \\
\mathrm{NH}_{3} \text {, followed by } \\
\text { a brief vacuum } \\
\text { anneal. }\end{array}$ & $\begin{array}{l}\text { Measurement of } \\
\text { anelastic strain } \\
\text { by elastic after- } \\
\text { effect. }\end{array}$ & $D_{T}$ & $285-310$ & 0.098 & 38,600 & $\begin{array}{l}\text { Effects of } \\
\text { possible inter- } \\
\text { stitial impurity } \\
\text { interactions were } \\
\text { not ascertained. }\end{array}$ & {$[6]$} \\
\hline $\mathrm{Nb} . .$. & N... & $\mathrm{Nb}, 99.9$ w/o & $\begin{array}{l}\mathrm{Nb} \text { foil; } \mathrm{N} \text { intro- } \\
\text { duced by anneal- } \\
\text { ing in air. }\end{array}$ & $\begin{array}{l}\text { Measurement of } \\
\text { anelastic strain } \\
\text { by internal } \\
\text { friction. }\end{array}$ & $D_{T}$ & $563-644$ & & 28,100 & $\begin{array}{l}\text { Effects of possible } \\
\text { interstitial impur- } \\
\text { ity interactions } \\
\text { were not ascer- } \\
\text { tained. }\end{array}$ & [7] \\
\hline $\mathrm{Nb} .$. & O......... & $\begin{array}{l}\text { Nb, } 99.4 \text { w/o; C, } \\
1000(\max .) ; \mathrm{Ta}, \\
5000 ; \mathrm{Ti}, 400 ; \\
\mathrm{Si}, 200 ; \mathrm{Fe}, 100 . \\
\text { Degassed to re- } \\
\text { move O and N } \\
\text { initially. }\end{array}$ & $\begin{array}{l}\mathrm{O} \text { introduced by } \\
\text { absorption of } \\
\text { molecular oxygen. }\end{array}$ & $\begin{array}{l}\text { Measurement of } \\
\text { anelastic strain } \\
\text { by elastic after- } \\
\text { effect and by } \\
\text { internal friction. }\end{array}$ & $D_{T}$ & $\begin{array}{c}44-150 \\
-\end{array}$ & 0.0212 & 29,910 & $\begin{array}{l}\text { Effect of inter- } \\
\text { stitial impurity } \\
\text { interactions were } \\
\text { determined. }\end{array}$ & [2] \\
\hline Nb....... & $0 \ldots \ldots$ & Not stated. & Not stated. & $\begin{array}{l}\text { Measurement of } \\
\text { anelastic strain } \\
\text { by elastic after- } \\
\text { effect. }\end{array}$ & $D_{T}$ & $148-168$ & 0.0147 & 27,600 & $\begin{array}{l}\text { Effects of } \\
\text { possible inter- } \\
\text { stitial impurity } \\
\text { interactions were } \\
\text { not ascertained. }\end{array}$ & [6] \\
\hline $\mathrm{Nb} . . \ldots \ldots \ldots$ & O............. & $\mathrm{Nb}, 99.9$ w/o & $\begin{array}{l}\text { Nb foil; } O \text { intro- } \\
\text { duced by anneal- } \\
\text { ing in air. }\end{array}$ & $\begin{array}{l}\text { Measurement of } \\
\text { anelastic strain } \\
\text { by internal } \\
\text { friction. }\end{array}$ & $D_{T}$ & $377-423$ & & 22,000 & $\begin{array}{l}\text { Effects of possible } \\
\text { interstitial impur- } \\
\text { ity interactions } \\
\text { were not ascer- } \\
\text { tained. }\end{array}$ & [7] \\
\hline $\mathrm{Nb} . \ldots \ldots .$. & $0 \ldots \ldots \ldots$ & $\begin{array}{l}\text { O, } 1000 ; \mathrm{N}, 500 \\
\quad \mathrm{C}, 300 \text {. }\end{array}$ & $\begin{array}{l}\mathrm{Nb} \text { cylinders ma- } \\
\text { chined from arc- } \\
\text { melted buttons; } \\
\text { reacted with } \mathrm{O}_{2} \\
\text { in modified } \\
\text { Sieverts appara- } \\
\text { tus; } \mathrm{O}_{2} \text { from de- } \\
\text { composition of } \\
\text { permanganate. }\end{array}$ & $\begin{array}{l}\text { Determination of } \\
\text { the concentra- } \\
\text { tion gradient by } \\
\text { microhardness } \\
\text { measure- } \\
\text { ments. }\end{array}$ & $\tilde{D}$ & $600-1000$ & $4.07 \times 10^{-3}$ & 24,900 & $\begin{array}{l}\text { The following were } \\
\text { assumed: (a) } \bar{D} \\
\text { independent of } \\
\text { concentration, } \\
\text { (b) Nb-Nb oxide } \\
\text { boundary sta- } \\
\text { tionary. }\end{array}$ & [8] \\
\hline $\mathrm{NbB}_{2} \ldots \ldots \ldots$ & B........... & Not stated. & $\begin{array}{l}\text { Boride layer was } \\
\text { formed on a Nb } \\
\text { cylinder by } \\
\text { boronizing in } \\
\text { boron carbide and } \\
\text { borax. }\end{array}$ & $\begin{array}{l}\text { Determination of } \\
\text { the concentra- } \\
\text { tion gradient by } \\
\text { microhardness } \\
\text { measurements. }\end{array}$ & $\widetilde{D}$ & $1100-1400$ & 2.94 & 59,000 & $\begin{array}{l}\widetilde{D} \text { was assumed to } \\
\text { be independent } \\
\text { of the B con- } \\
\text { centration. }\end{array}$ & [9] \\
\hline $\mathrm{NbC} . \ldots \ldots \ldots$ & C............ & Not stated. & $\begin{array}{l}\text { Niobium sheets } \\
\text { heated in pres- } \\
\text { ence of } \mathrm{CH}_{4}-\mathrm{Ar} \\
\text { atmosphere. }\end{array}$ & $\begin{array}{l}\text { Rate of carbide } \\
\text { layer growth } \\
\text { measured with } \\
\text { filar micro- } \\
\text { meter. }\end{array}$ & $\widetilde{D}$ & $1900-2300$ & 1.2 & 79,000 & $\begin{array}{l}\text { The results } \\
\text { represent average } \\
\text { values for } \widetilde{D} \text { over } \\
\text { the composition } \\
\text { range. } \tilde{D} \text { was } \\
\text { assumed to be } \\
\text { independent of } \\
\text { concentration. } \\
\text { The boundary } \\
\text { compositions of } \\
\text { the carbide layer } \\
\text { were taken from } \\
\text { phase equilibrium } \\
\text { diagrams [11]. }\end{array}$ & [10] \\
\hline NbC....... & C.... & $\begin{array}{l}\text { Nb slab; W, } 290 ; \\
\text { Fe <100; C, } 100 ; \\
\text { Si, 50; W, 110; } \\
\text { Ta, 600; Zr < } 100 .\end{array}$ & $\begin{array}{l}\text { Niobium slabs } \\
\text { packed in high- } \\
\text { purity lampblack } \\
\text { and heated. }\end{array}$ & $\begin{array}{l}\text { Rate of carbide } \\
\text { layer growth; } \\
\text { movement of inert } \\
\text { markers (holes) } \\
\text { in diffusion } \\
\text { regions of bonded } \\
\text { graphite and } \mathrm{Nb} \\
\text { slabs. }\end{array}$ & $\tilde{D}$ & $1700-2300$ & 7.6 & 88,200 & $\begin{array}{l}\text { Nb was found to } \\
\text { be immobile as } \\
\text { compared to C. } \\
\widetilde{D} \text { was assumed to } \\
\text { be independent } \\
\text { of concentration. } \\
\text { X-ray data indi- } \\
\text { cated higher } \\
\text { diffusion rates } \\
\text { in the more car- } \\
\text { bon deficient } \\
\text { region of the } \\
\text { NbC layer. }\end{array}$ & {$[12,13]$} \\
\hline
\end{tabular}

${ }^{\text {a }}$ Impurity concentrations are given in ppm unless otherwise stated. 
Niobium-Continued

\begin{tabular}{|c|c|c|c|c|c|c|c|c|c|c|}
\hline $\begin{array}{l}\text { Solvent } \\
\text { medium }\end{array}$ & $\begin{array}{l}\text { Diffusing } \\
\text { element }\end{array}$ & Purity ${ }^{a}$ & $\begin{array}{l}\text { Preparation and } \\
\text { properties }\end{array}$ & Method & $\begin{array}{c}\text { Diffusion } \\
\text { coefficient }\end{array}$ & $\begin{array}{c}\text { Temperature } \\
\text { range, }{ }^{\circ} \mathrm{C}\end{array}$ & $D_{0} \mathrm{~cm}^{2} / \mathrm{s}$ & $Q \mathrm{cal} / \mathrm{mol}$ & Comments & Ref. \\
\hline $\mathrm{NbC}_{0.98} \ldots .$. & C... & Not stated. & $\begin{array}{l}\text { Nb carbides were } \\
\text { synthesized from } \\
\mathrm{Nb}_{2} \mathrm{O}_{5} .\end{array}$ & $\begin{array}{l}\text { Measurement of } \\
\text { attenuation of } \\
\beta \text {-radiation by } \\
\text { the method of } \\
\text { Gruzin [5] (ratio } \\
\text { of activities). }\end{array}$ & $\begin{array}{l}D^{*} \text { (self- } \\
\quad \text { diffusion) }\end{array}$ & $1600-2000$ & $1.0 \times 10^{-6}$ & 32,300 & $\begin{array}{l}\text { High diffusivity } \\
\text { paths probably } \\
\text { existed along } \\
\text { pores. }\end{array}$ & [4] \\
\hline $\mathrm{NbC}_{0.98} \ldots$ & $\mathrm{Nb} .$. & Not stated. & $\begin{array}{l}\text { Nb carbides were } \\
\text { synthesized from } \\
\mathrm{Nb}_{2} \mathrm{O}_{5} .\end{array}$ & $\begin{array}{l}\text { Measurement of } \\
\text { attenuation of } \\
\beta \text {-radiation by } \\
\text { the method of } \\
\text { Gruzin [5] (ratio } \\
\text { of activities). }\end{array}$ & $D^{*}$ & $1800-2200$ & $5.5 \times 10^{-6}$ & 55,000 & $\begin{array}{l}\text { High diffusivity } \\
\text { paths probably } \\
\text { existed along } \\
\text { pores. }\end{array}$ & [4] \\
\hline $\mathrm{Nb}_{2} \mathrm{C} \ldots \ldots$ & C............... & Not stated. & $\begin{array}{l}\mathrm{Nb} \text { carbides were } \\
\text { synthesized from } \\
\mathrm{Nb}_{2} \mathrm{O}_{5} .\end{array}$ & $\begin{array}{l}\text { Measurement of } \\
\text { attenuation of } \\
\beta \text {-radiation by } \\
\text { the method of } \\
\text { Gruzin [5] (ratio } \\
\text { of activities). }\end{array}$ & $D^{*}$ & $1600-2000$ & $5.7 \times 10^{-6}$ & 29,800 & $\begin{array}{l}\text { High diffusivity } \\
\text { paths probably } \\
\text { existed along } \\
\text { pores. }\end{array}$ & [4] \\
\hline $\mathrm{Nb}_{4} \mathrm{C}_{3} \ldots \ldots$ & C... & Not stated. & $\begin{array}{l}\mathrm{Nb} \text { carbides were } \\
\text { synthesized from } \\
\mathrm{Nb}_{2} \mathrm{O}_{5} .\end{array}$ & $\begin{array}{l}\text { Measurement of } \\
\text { attenuation of } \\
\beta \text {-radiation by } \\
\text { the method of } \\
\text { Gruzin [5] (ratio } \\
\text { of activities). }\end{array}$ & $D^{*}$ & $1600-2000$ & $8.8 \times 10^{-6}$ & 35,300 & $\begin{array}{l}\text { High diffusivity } \\
\text { paths probably } \\
\text { existed along } \\
\text { pores. }\end{array}$ & [4] \\
\hline NbC:TiC... & $\mathrm{Nb} . .$. & $\begin{array}{l}\text { Starting ma- } \\
\text { terials: } \mathrm{TiO}_{2} \\
99.7 \text { w/o; Nb } 98 \\
\text { w/o; lamp black } \\
\text { with } 4 \text { w/o ash } \\
\text { residue. }\end{array}$ & $\begin{array}{l}\mathrm{TiO}_{2}-\mathrm{Nb} \text { mixture } \\
\text { carbidized with } \\
\text { lamp black in } \\
\text { graphite furnace, } \\
\text { carbide powder } \\
\text { pressed and } \\
\text { sintered; density } \\
6.55 \mathrm{~g} / \mathrm{cm}^{3} ; \text { grain } \\
\text { size } 20-30 \mu \mathrm{m} .\end{array}$ & $\begin{array}{l}\text { Measurement of } \\
\text { attenuation of } \\
\beta \text {-radiation by } \\
\text { the method of } \\
\text { Zhukhovitskii } \\
\text { and Geodakyan } \\
\text { [15] (nonexponen- } \\
\text { tial absorption). }\end{array}$ & $D^{*}$ & $2170-2280$ & 470 & 120,000 & & [14] \\
\hline $\mathrm{Nb}_{2} \mathrm{O}_{4.978 \ldots} .$. & $0 \ldots \ldots \ldots$ & $\begin{array}{c}\mathrm{Ta}_{2} \mathrm{O}_{5}, 0.01 \mathrm{w} / \mathrm{o} \\
\mathrm{SiO}_{2}, 0.02 \mathrm{w} / \mathrm{o} \\
\mathrm{TiO}_{2}, 0.005 \mathrm{w} / \mathrm{o} \\
\mathrm{Fe}_{2} \mathrm{O}_{3}, 0.003 \text { w/o. }\end{array}$ & $\begin{array}{l}\text { Hot-pressed anion- } \\
\text { deficient } \\
\text { oxide slabs, } \\
\text { density of } 4.55 \\
\mathrm{~g} / \mathrm{cm}^{3} \text {. }\end{array}$ & $\begin{array}{l}\text { Microphoto- } \\
\text { metric determina- } \\
\text { tion of } \\
\text { the color change } \\
\text { associated with } \\
\text { the advance of } \\
\text { an "oxygen-rich" } \\
\text { front into an } \\
\text { oxygen deficient } \\
\text { oxide slab. }\end{array}$ & $\tilde{D}$ & $500-900$ & 0.038 & 28,200 & $\begin{array}{c}\widetilde{D} \text { was measured for } \\
\text { the particular oxide } \\
\text { composition given. }\end{array}$ & [16] \\
\hline $\begin{array}{l}\mathrm{Nb}_{2} \mathrm{O}_{5}+ \\
\quad 1 \mathrm{~m} / \mathrm{o} \\
\mathrm{ZrO}_{2}\end{array}$ & 0 & $\begin{array}{l}\text { Impurity content } \\
\text { of } \mathrm{Nb}_{2} \mathrm{O}_{5} \text { same as } \\
\text { given above. }\end{array}$ & $\begin{array}{l}\text { Hot-pressed anion- } \\
\text { deficient oxide } \\
\text { slabs, densities } \\
\text { varied between } \\
4.52 \text { and } 4.55 \\
\mathrm{~g} / \mathrm{cm}^{3} \text {. }\end{array}$ & $\begin{array}{l}\text { Microphotometric } \\
\text { determination of } \\
\text { the color change } \\
\text { associated with } \\
\text { the advance of an } \\
\text { "oxygen-rich" } \\
\text { front into an } \\
\text { oxygen-deficient } \\
\text { oxide slab. }\end{array}$ & $\tilde{D}$ & $500-900$ & 0.00201 & 24,000 & & [16] \\
\hline $\begin{array}{c}\mathrm{Nb}_{2} \mathrm{O}_{5}+ \\
1 \mathrm{~m} / \mathrm{o} \\
\mathrm{TiO}_{2}\end{array}$ & $0 \ldots$ & $\begin{array}{l}\text { Impurity content } \\
\text { of } \mathrm{Nb}_{2} \mathrm{O}_{5} \text { same as } \\
\text { given above. }\end{array}$ & $\begin{array}{l}\text { Hot-pressed anion- } \\
\text { deficient oxide } \\
\text { slabs, densities } \\
\text { varied between } \\
4.52 \text { and } 4.55 \\
\mathrm{~g} / \mathrm{cm}^{3} \text {. }\end{array}$ & $\begin{array}{l}\text { Microphotometric } \\
\text { determination of } \\
\text { the color change } \\
\text { associated with } \\
\text { the advance of an } \\
\text { "oxygen-rich" } \\
\text { front into an } \\
\text { oxygen-deficient } \\
\text { oxide slab. }\end{array}$ & $D$ & $500-900$ & 0.0262 & 30,700 & & [16] \\
\hline $\begin{array}{l}\mathrm{Nb}_{2} \mathrm{O}_{5}+ \\
\quad 1 \mathrm{~m} / \mathrm{o} \\
\mathrm{V}_{2} \mathrm{O}_{5}\end{array}$ & O... & $\begin{array}{l}\text { Impurity content } \\
\text { of } \mathrm{Nb}_{2} \mathrm{O}_{5} \text { same as } \\
\text { given above. }\end{array}$ & $\begin{array}{l}\text { Hot-pressed anion- } \\
\text { deficient oxide } \\
\text { slabs, densities } \\
\text { varied between } \\
4.52 \text { and } 4.55 \\
\text { g/cm } 1 \mathrm{~cm}^{3} \text {. }\end{array}$ & $\begin{array}{l}\text { Microphotometric } \\
\text { determination of } \\
\text { the color change } \\
\text { associated with } \\
\text { the advance of an } \\
\text { "oxygen-rich" } \\
\text { front into an } \\
\text { oxygen-deficient } \\
\text { oxide slab. }\end{array}$ & $\tilde{D}$ & $500-900$ & 0.0432 & 32,400 & & [16] \\
\hline
\end{tabular}

a Impurity concentrations are given in ppm unless otherwise stated.

[1] C. A. Wert, J. Appl. Phys. 21, 1196 (1950).

[2] R. W. Powers and M. V. Doyle, J. Appl. Phys. 30, 514 (1959).

[3] ibid. Trans. AIME 209, 1285 (1957).

[4] P. V. Gel'd and V. D. Lyubimov, Isv. Akad. Nauk SSSR, Otdel. Tekh. Nauk Met. I Toplivo [6], 199 (1961).

[5] P. L. Gruzin, Dokl. Akad. Nauk SSSR 86, 289 (1952).

[6] C. Y. Ang. Acta Met. 1, 123 (1953).

[7] J. W. Marx, G. S. Baker and J. M. Silvertsen, Acta Met. 1, 193 (1953).

[8] W. D. Klopp, C. T. Sims, and R. I. Jaffe, Trans, ASM 51, 282 (1959).

[9] G. V. Samsonov and A. P. Epik, Dopovidi Akad. Nauk Ukr. RSR [1], 67 (1964).
[10] R. Resnick, R. Steinitz and L. Seigle, Trans. AIME 223, 1915 (1965)

[11] E. K. Storms and N. H. Krikorian, J. Phys. Chem. 64, 1471 (1960).

[12] W. F. Brizes, L. H. Cadoff and J. M. Tobin, J. Nucl. Mater. 20, 57 (1966).

[13] J. M. Tobin, L. M. Adelsberg, L. H. Cadoff and W. F. Brizes, Nuclear applications of nonfissionable ceramics, p. 257, American Nuclear Society and American Ceramic Society (American Nuclear Society, Inc., Hinsdale, Ill., 1966).

[14] M. L. Baskin, V. I. Tret'yakov, and I. N. Chaporova, Fiz. Metal. I Metailovid. 12, 860 (1961).

[15] A. A. Zhukhovitskii and V. A. Geodakyan, Dokl. Akad. Nauk SSSR 102, 301 (1955).

[16] D. L. Douglass, Corrosion of Reactor Materials, 2, p. 233 (International Atomic Energy Agency, Vienna, Austria, 1962). 
Tantalum

\begin{tabular}{|c|c|c|c|c|c|c|c|c|c|}
\hline $\begin{array}{l}\text { Solvent } \\
\text { medium }\end{array}$ & $\begin{array}{l}\text { Diffusing } \\
\text { element }\end{array}$ & Purity ${ }^{a}$ & $\begin{array}{l}\text { Preparation and } \\
\text { properties }\end{array}$ & Method & $\begin{array}{l}\text { Diffusion } \\
\text { coefficient }\end{array}$ & $\begin{array}{c}\text { Temperature } \\
\text { range, }{ }^{\circ} \mathrm{C}\end{array}$ & $D_{0} \mathrm{~cm}^{2} / \mathrm{s}$ & $Q \mathrm{cal} / \mathrm{mol}$ & Comments \\
\hline $\mathrm{Ta} \ldots \ldots \ldots . .$. & C........... & Not stated. & Not stated. & $\begin{array}{l}\text { Measurement of } \\
\text { anelastic strain } \\
\text { by after-effect } \\
\text { and by internal } \\
\text { friction. }\end{array}$ & $\begin{array}{l}D_{T} \text { (tracer } \\
\quad \text { diffusion) }\end{array}$ & $55-160$ & 0.015 & 27,000 & $\begin{array}{l}\text { Effects of } \\
\text { possible inter- } \\
\text { stitial impurity } \\
(\mathrm{C}, \mathrm{N}, \mathrm{O}) \text { inter- } \\
\text { actions were not } \\
\text { ascertained. }\end{array}$ \\
\hline Та.......... & C......... & $\begin{array}{l}\text { Metallic con- } \\
\quad \text { taminants not } \\
\text { stated; } 99.9 \mathrm{w} / \mathrm{o} \\
\text { Ta; C, } 100-900 .\end{array}$ & $\begin{array}{l}\mathrm{C} \text { was added to a } \\
\text { degassed wire by } \\
\text { pyrolysis of } \\
\text { carbonaceous va- } \\
\text { por or by heating a } \\
\text { wire coated with } \\
\text { a carbon black- } \\
\text { water slurry. }\end{array}$ & $\begin{array}{l}\text { Measurement of } \\
\text { anelastic strain } \\
\text { by elastic after- } \\
\text { effect and by } \\
\text { internal friction. }\end{array}$ & $D_{T}$ & $193-350$ & $6.1 \times 10^{-3}$ & 38,510 & $\begin{array}{l}\text { Effects of inter- } \\
\text { stitial impurity } \\
\text { interactions were } \\
\text { determined; carbide } \\
\text { precipitation was } \\
\text { observed at "high" } \\
\text { C concentrations. }\end{array}$ \\
\hline Та...... & C.... & C, 1000 . & $\begin{array}{l}{ }^{14} \mathrm{C} \text { was introduced } \\
\text { into about } 10 \mathrm{~mm} \\
\text { center portion of } \\
\text { a } 70 \mathrm{~mm} \mathrm{Ta} \text { wire. }\end{array}$ & $\begin{array}{l}\text { Determination of } \\
\text { the concentration } \\
\text { gradient by } \\
\text { radioisotope } \\
\text { distribution. }\end{array}$ & $D_{T}$ & $600-2600$ & $2.78 \times 10^{-3}$ & 24,600 & $\begin{array}{l}\text { An increase in the } \\
\text { activation energy } \\
\text { was observed below } \\
800{ }^{\circ} \mathrm{C} \text {. }\end{array}$ \\
\hline $\mathrm{Ta} \ldots .$. & $\mathrm{N} \ldots$ & $\begin{array}{l}\text { Metallic con- } \\
\text { taminants not } \\
\text { stated; } 99.9 \mathrm{w} / \mathrm{o} \\
\text { Ta; O, 320; N, } \\
\text { 50-1000. }\end{array}$ & $\begin{array}{l}\text { Degassed wire was } \\
\text { enriched in } \mathrm{N} \text { by } \\
\text { heating in } \mathrm{N}_{2} \text { or } \\
\mathrm{NH}_{3} \text { at low partial } \\
\text { pressure. }\end{array}$ & $\begin{array}{l}\text { Measurement of } \\
\text { anelastic strain } \\
\text { by elastic after- } \\
\text { effect and by } \\
\text { internal friction. }\end{array}$ & $D_{T}$ & $187-350$ & $5.6 \times 10^{-3}$ & 37,840 & $\begin{array}{l}\text { Effects of inter- } \\
\text { stitial impurity } \\
\text { were determined. }\end{array}$ \\
\hline Та....... & N.... & Not stated. & $\begin{array}{l}\mathrm{N} \text { introduced by } \\
\text { heating a wire in } \\
\mathrm{NH}_{3} \text {, followed by } \\
\text { a brief vacuum } \\
\text { anneal. }\end{array}$ & $\begin{array}{l}\text { Measurement of } \\
\text { anelastic strain } \\
\text { by elastic after- } \\
\text { effect and by } \\
\text { internal friction. }\end{array}$ & $D_{T}$ & $360-662$ & 0.0123 & 39,800 & $\begin{array}{l}\text { Effects of } \\
\text { possible inter- } \\
\text { stitial impurity } \\
\text { interactions were } \\
\text { not ascertained. }\end{array}$ \\
\hline Ta.... & N... & $\begin{array}{l}\mathrm{C}, 60 ; \mathrm{N}<20 \\
\text { W }, 1000 ; \mathrm{Cu} \\
<40 ; \mathrm{Fe}<50 \\
\mathrm{Si}<100 ; \mathrm{Ti} \\
<50 ; \mathrm{Mo}<80 ; \\
\mathrm{Sn}<40 ; \mathrm{Nb}, 500 ; \\
\text { O, } 60 ; \text { all others } \\
<20 \text {. }\end{array}$ & $\begin{array}{l}\text { Cold-rolled } \\
\text { specimens were } \\
\text { polished, anneal- } \\
\text { ed, etched; } \\
\text { reacted with } \mathrm{N}_{2} \\
(99.995 \mathrm{v} / \mathrm{o}) .\end{array}$ & $\begin{array}{l}\text { Determination of } \\
\text { the concentration } \\
\text { gradient by } \\
\text { microhardness } \\
\text { measurements. }\end{array}$ & $\begin{array}{c}\tilde{D} \text { (chemical } \\
\text { diffusion) }\end{array}$ & $800-1000$ & 0.024 & 40,900 & $\begin{array}{l}\text { Linear dependence } \\
\text { of hardness on } \mathrm{N} \\
\text { concentration was } \\
\text { demonstrated by } \\
\text { Gebhardt et al. [13]; } \\
\widetilde{D} \text { was assumed to } \\
\text { be independent of } \\
\text { the } \mathrm{N} \text { concentra- } \\
\text { tion. }\end{array}$ \\
\hline Та....... & N.... & $\begin{array}{l}\mathrm{O}, 16 ; \mathrm{N}, 10 ; \mathrm{C}, \\
30 ; \mathrm{Nb}, 100-300 ; \\
\mathrm{Cu}, 30 ; \text { all } \\
\text { others }<10 .\end{array}$ & $\begin{array}{l}\text { Ta plates were } \\
\text { used below } 1000 \\
{ }^{\circ} \mathrm{C} \text {, cylindrical } \\
\text { specimens were } \\
\text { used above } 1000 \\
\text { 'C: vacuum an- } \\
\text { nealed; reacted } \\
\text { with prepurified } \\
\mathrm{N}_{2}(99.996 \mathrm{v} / \mathrm{o}) .\end{array}$ & $\begin{array}{l}\text { Determination of } \\
\text { concentration } \\
\text { gradient by } \\
\text { microhardness } \\
\text { measurements. }\end{array}$ & $\widetilde{D}$ & $800-1300$ & 0.0182 & 41,100 & $\begin{array}{l}\widetilde{D} \text { was assumed to } \\
\text { be independent of } \\
\text { the } N \text { concentra- } \\
\text { tion. }\end{array}$ \\
\hline Ta.......... & $0 \ldots$ & $\begin{array}{l}\text { Metallic contam- } \\
\text { inants not stated; } \\
99.9 \text { w/o Ta; O, } \\
90-1630 ; \mathrm{N}, 50\end{array}$ & $\begin{array}{l}\text { Degassed Ta wire } \\
\text { loaded with } \mathrm{O} \text { by } \\
\text { heating in } \mathrm{O}_{2} \text { at } \\
\text { low partial } \\
\text { pressure. }\end{array}$ & $\begin{array}{l}\text { Measurement of } \\
\text { anelastic strain } \\
\text { by elastic after- } \\
\text { effect and by } \\
\text { internal friction. }\end{array}$ & $D_{T}$ & $38-250$ & $4.4 \times 10^{-3}$ & 25,450 & $\begin{array}{l}\text { Effects of inter- } \\
\text { stitial impurity } \\
\text { interactions were } \\
\text { determined. }\end{array}$ \\
\hline Ta... & $0 \ldots$ & Not stated. & Not stated. & $\begin{array}{l}\text { Measurement of } \\
\text { anelastic strain } \\
\text { by elastic after- } \\
\text { effect and by } \\
\text { internal friction. }\end{array}$ & $D_{T}$ & $155-355$ & 0.0190 & 27,300 & $\begin{array}{l}\text { Effects of } \\
\text { possible inter- } \\
\text { stitial impurity } \\
\text { interactions were } \\
\text { not ascertained. }\end{array}$ \\
\hline Ta..... & $0 \ldots \ldots$ & Not stated. & $\begin{array}{l}\text { Band-shaped } \\
\text { specimens with } \\
\text { one section } \\
\text { enriched in O } \\
\text { used. }\end{array}$ & $\begin{array}{l}\text { Determination of } \\
\text { the concentra- } \\
\text { tion gradient by } \\
\text { microhardness } \\
\text { measurements. }\end{array}$ & $\bar{D}$ & $700-1400$ & 0.015 & 26,700 & $\begin{array}{l}\text { Linear dependence } \\
\text { of hardness on } \mathrm{O} \\
\text { concentration was } \\
\text { demonstrated up } \\
\text { to } 1.1 \text { at. } \% \mathrm{O} \text {. } \\
D \text { was assumed to } \\
\text { be independent of } \\
\text { the } \mathrm{O} \text { concentra- } \\
\text { tion. }\end{array}$ \\
\hline Та..... & $0 \ldots$ & $\begin{array}{l}\mathrm{O}, 16 ; \mathrm{N}, 10 ; \mathrm{C}, \\
30 ; \mathrm{Nb}, 100-300 ; \\
\mathrm{Cu}, 30 ; \text { all } \\
\text { others < } 10 .\end{array}$ & $\begin{array}{l}\text { Ta plates were } \\
\text { used below } 1000 \\
{ }^{\circ} \mathrm{C} \text {, cylindrical } \\
\text { specimens were } \\
\text { used above } 1000 \\
{ }^{\circ} \mathrm{C} \text {; vacuum an- } \\
\text { nealed; reacted } \\
\text { with } \mathrm{O}_{2} \text { from } \\
\text { decomposition of } \\
\text { permanganate. }\end{array}$ & $\begin{array}{l}\text { Determination of } \\
\text { the concentra- } \\
\text { tion gradient by } \\
\text { microhardness } \\
\text { measurements. }\end{array}$ & $\widetilde{D}$ & $900-1200$ & $2.14 \times 10^{-3}$ & 22,900 & $\begin{array}{l}\text { Linear dependence } \\
\text { of hardness on } \mathrm{O} \\
\text { concentration was } \\
\text { established at low } \\
\text { concentrations by } \\
{[8] . D \text { was }} \\
\text { assumed to be in- } \\
\text { dependent of the } \\
O \text { concentration. }\end{array}$ \\
\hline $\mathrm{TaB}_{2}$. & B..... & Not stated. & $\begin{array}{l}\text { Boride layer was } \\
\text { formed on a Ta } \\
\text { cylinder by boro- } \\
\text { nizing in boron } \\
\text { carbide and borax. }\end{array}$ & $\begin{array}{l}\text { Determination of } \\
\text { the concentra- } \\
\text { tion gradient by } \\
\text { microhardness } \\
\text { measurements. }\end{array}$ & $\widetilde{D}$ & $1100-1400$ & $9.44 \times 10^{-4}$ & 47,000 & $\begin{array}{l}D \text { was assumed to } \\
\text { be independent of } \\
\text { the } \mathrm{B} \text { concen- } \\
\text { tration. }\end{array}$ \\
\hline
\end{tabular}


Tantalum-Continued

\begin{tabular}{|c|c|c|c|c|c|c|c|c|c|c|}
\hline $\begin{array}{l}\text { Solvent } \\
\text { medium }\end{array}$ & $\begin{array}{l}\text { Diffusing } \\
\text { element }\end{array}$ & Purity $^{a}$ & $\begin{array}{l}\text { Preparation and } \\
\text { properties }\end{array}$ & Method & $\begin{array}{c}\text { Diffusion } \\
\text { coefficient }\end{array}$ & $\begin{array}{c}\text { Temperature } \\
\text { range, }{ }^{\circ} \mathrm{C}\end{array}$ & $D_{0} \mathrm{~cm}^{2} / \mathrm{s}$ & $Q \mathrm{cal} / \mathrm{mol}$ & Comments & Ref. \\
\hline TaC... & C............. & $\begin{array}{l}\mathrm{Nb}, 40-50 ; \\
\mathrm{Ti},<25 ; \\
\mathrm{Mo},<40 ; \\
\mathrm{W}<40 ; \text { all } \\
\text { others }<50 ; \mathrm{O}_{2} \\
\text { and } \mathrm{N}_{2} \sim 100 \text { in } \\
\text { carbide slab. }\end{array}$ & $\begin{array}{l}\text { Slabs with marker } \\
\text { holes near the } \\
\text { surface were car- } \\
\text { burized in high } \\
\text { purity graphite } \\
\text { powder, for } \\
\text { Kirckendall shift } \\
\text { measurements. } \\
\text { TaC slabs with low } \\
\text { carbon content } \\
\text { (45 to } 46 \text { at pct } \\
\text { C) were heated in } \\
\text { CH } 4 \text {-Ar mixture for } \\
\text { chemical diffusion } \\
\text { measurements. }\end{array}$ & \begin{tabular}{|l} 
Determination of \\
carbon concentra- \\
tion profile by \\
x-ray diffrac- \\
tion analysis; \\
A Matano analysis \\
was performed, \\
but values of $D_{o}$ \\
and $Q$ were cal- \\
culated from \\
values of $D$ \\
averaged over \\
the slab.
\end{tabular} & $\tilde{D}$ & $1700-2700$ & 0.18 & 85,000 & $\begin{array}{l}\text { The marker move- } \\
\text { ment study indi- } \\
\text { cated that } \\
D_{c}: D_{\mathrm{Ta}} \cong 80: 1 \\
\text { in } \mathrm{TaC} \text { at } 2500^{\circ} \mathrm{C} \text {. } \\
\text { The Matano anal- } \\
\text { ysis indicated } \\
\text { that } \bar{D} \text { had a com- } \\
\text { plicated dependence } \\
\text { on C concentration. } \\
\text { Grain boundary } \\
\text { diffusion was not } \\
\text { important. }\end{array}$ & [10] \\
\hline $\mathrm{TaC} \ldots \ldots$ & C................ & Not stated. & & $\begin{array}{l}\text { Determination of } \\
\text { carbon concentra- } \\
\text { tion profiles by } \\
\text { electron micro- } \\
\text { probe analysis. }\end{array}$ & $D$ & $2100-2650$ & 1.8 & 87,000 & $\begin{array}{l}\text { The carbon concen- } \\
\text { tration profiles } \\
\text { were found to be } \\
\text { nonlinear in } \\
\text { the } \mathrm{TaC} \text { layer. }\end{array}$ & [11] \\
\hline $\mathrm{TaC}$. & C.... & $\begin{array}{l}\mathrm{Nb}, 50 ; \mathrm{Ti}<25 \\
\mathrm{Fe}, 10 ; \mathrm{Mo}<50 \text {; } \\
\text { W }<50 ; \text { all } \\
\text { other metals } \\
<50 ; \mathrm{O}, 15-75 ; \\
\mathrm{N}, 10-30 ; \mathrm{C} \text {; } \\
\text { 10-30. }\end{array}$ & $\begin{array}{l}\text { Carbide layer was } \\
\text { formed by heat- } \\
\text { ing Ta sheets in } \\
\text { an Ar-methane at- } \\
\text { mosphere. }\end{array}$ & $\begin{array}{l}\text { Measurement of } \\
\text { the rate of move- } \\
\text { ment of the } \mathrm{TaC} \text { - } \\
\mathrm{Ta}_{2} \mathrm{C} \text { and } \mathrm{Ta}_{2} \mathrm{C}- \\
\mathrm{Ta} \text { interfaces. }\end{array}$ & $\tilde{D}$ & $1800-2706$ & 1.04 & 86,000 & $\begin{array}{l}\text { Rate of movement } \\
\text { of the interfaces } \\
\text { was assumed to be } \\
\text { controlled by } \\
\text { diffusion through } \\
\text { the carbide layers. } \\
D \text { was assumed to } \\
\text { be independent of } \\
\text { the C concen- } \\
\text { tration. }\end{array}$ & [12] \\
\hline TaC.. & W..... & $\begin{array}{l}\text { Free C, } 500 ; \\
\quad \text { Fe, } 800 .\end{array}$ & $\begin{array}{l}\text { Carbide was formed } \\
\text { by carburization } \\
\text { in graphite } \\
\text { charge; carbide } \\
\text { powder sintered; } \\
\text { density } 14.2 \mathrm{~g} / \mathrm{cm}^{3} ; \\
\text { porosity } 3 \% \text {; } \\
\text { grain size } \\
50-100 \mu \mathrm{m} \text {. }\end{array}$ & $\begin{array}{l}\text { Measurement of } \\
\text { the attenuation } \\
\text { of the } \beta \text {-radia- } \\
\text { tion by the } \\
\text { method of Kryukov } \\
\text { and Zhukhovitskii } \\
{[14] \text {. }}\end{array}$ & $\begin{array}{l}D^{*} \text { (self- } \\
\text { diffusion) }\end{array}$ & $2130-2300$ & 19.2 & 142,000 & $\begin{array}{l}\text { There may be some } \\
\text { error in this } \\
\text { data due to vapor- } \\
\text { ization of the } \\
\text { radioactive layer. }\end{array}$ & [13] \\
\hline $\mathrm{Ta}_{2} \mathrm{C}$. & C..... & $\mid \begin{array}{c}\mathrm{Nb}, 50 ; \mathrm{Ti}<25 \\
\mathrm{Fe}, 10 ; \mathrm{Mo}<50 \\
\mathrm{~W}<50 ; \text { all } \\
\text { other metals }<50 \\
\mathrm{O}, 15-75 ; \mathrm{N} \\
10-30 ; \mathrm{C}, 10-30\end{array}$ & $\begin{array}{l}\text { Carbide layer was } \\
\text { formed by heat- } \\
\text { ing Ta sheets in } \\
\text { Ar-methane } \\
\text { atmosphere. }\end{array}$ & $\begin{array}{l}\text { Measurement of } \\
\text { the rate of move- } \\
\text { ment of } \mathrm{TaC}-\mathrm{Ta}_{2} \mathrm{C} \\
\text { and } \mathrm{Ta} \mathrm{a}_{2} \mathrm{C}-\mathrm{Ta} \text { in- } \\
\text { faces. }\end{array}$ & $\bar{D}$ & $1800-2700$ & 7.0 & 89,000 & $\begin{array}{l}\text { Rate of movement } \\
\text { of the interfaces } \\
\text { was assumed to be } \\
\text { controlled by } \\
\text { diffusion through } \\
\text { the carbide layers. } \\
D \text { was assumed to } \\
\text { be independent of } \\
\text { C concentration. }\end{array}$ & [15] \\
\hline
\end{tabular}

${ }^{\text {a }}$ Impurity concentrations are given in ppm unless otherwise stated.

[1] C. A. Wert, J. Appl. Phys. 21, 1196 (1950)

[2] R. W. Powers and M. V. Doyle, J. Appl. Phys. 28, 255 (1957); ibid 30, 514 (1959).

[3] D. F. Kalinovich, I. I. Kovenskiy, and M. D. Smolin, Fiz. Metal. I Metalloved. 18, 314 (1964).

[4] C. Y. Ang. Acta Met. 1, 123 (1953).

[5] K. Östhagen and P. Kofstad, J. Less-Common Metals 5, 7 (1963).

[6] W. M. Albrecht, W. D. Ǩloop, B. G. Koehl, and R. I. Jaffe, Trans. AIME 22 1, 110 (1961).

[7] E. Gebhardt, H. D. Seghezzi, and A. Stegherr, Z. Metallk. 48, 624 (1957)

8] R. H. Perkins, U.S. At. Energy Comm. Report LA-2136 (1957)

[9] G. V. Samsonov and A. P. Epik, Dopovidi Akad. Nauk Ukr. RSR [1], 67 (1964).

[10] R. Resnick and L. Seigle, Trans. AIME 236, 1732 (1966).

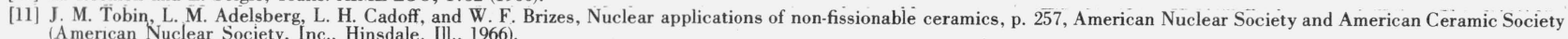

[12] R. Resnick, R. Steinitz, and L. Steigle, Trans. AIME 233, 1915 (1965).

[13] M. S. Baskin, V. I. Tret'yakov, and I. N. Chaporova, Fiz. Metal. I Metalloved. 14, 422 (1962).

[14] A. A. Zhukhovitskii and S. N. Kryikov, Dokl. Akad. Nauk SSSR 102, 301 (1955).

[15] E. Gebhardt, H. D. Seghezzi, and W. Dürrschnabel, Plansee Proc. 3rd Plansee Seminar, Reutte, Austria, p. 291 (1958).

Thorium

\begin{tabular}{|c|c|c|c|c|c|c|c|c|c|c|}
\hline $\begin{array}{l}\text { Solvent } \\
\text { medium }\end{array}$ & $\begin{array}{l}\text { Diffusing } \\
\text { element }\end{array}$ & Purity ${ }^{a}$ & $\begin{array}{l}\text { Preparation and } \\
\text { properties }\end{array}$ & Method & $\begin{array}{l}\text { Diffusion } \\
\text { coefficient }\end{array}$ & $\begin{array}{c}\text { Temperature } \\
\text { range, },{ }^{\circ} \mathrm{C}\end{array}$ & $D_{0} \mathrm{~cm}^{2} / \mathrm{s}$ & $Q \mathrm{cal} / \mathrm{mol}$ & Comments & Ref. \\
\hline Th..... & C, 0,4 w/o. & Not stated. & $\begin{array}{l}\text { Thorium cylinders } \\
\text { were carburized in } \\
\text { a graphite } \\
\text { charge. }\end{array}$ & $\begin{array}{l}\text { Determination of } \\
\text { the concentra- } \\
\text { tion gradient } \\
\text { by chemical } \\
\text { analysis. }\end{array}$ & 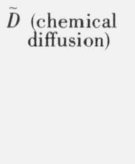 & $1000-1200$ & $\sim 0.03$ & 38,200 & $\begin{array}{l}\text { The concentration } \\
\text { dependence of } D \\
\text { was determined by } \\
\text { the method of } \\
\text { Smith and Daniel- } \\
\text { son [2]; } D_{0} \text { has a } \\
\text { large uncertainty. }\end{array}$ & {$[1]$} \\
\hline $\mathrm{ThO}_{2} \ldots \ldots$ & Ce......... & Not stated. & $\begin{array}{l}\text { Grain size } 0.2 \mu \mathrm{m} \text {; } \\
\text { irradiated with } \\
3.8 \times 10^{17} \mathrm{fast} \\
\text { neutrons } / \mathrm{cm}^{2} .\end{array}$ & $\begin{array}{l}\text { Determination of } \\
\text { the rate of } \\
\text { leaching of } \\
\text { fission prod- } \\
\text { ucts into molten } \\
\mathrm{NaCl} \text {. }\end{array}$ & $\begin{array}{c}D_{T} \text { (tracer } \\
\text { diffusion) }\end{array}$ & $910-1010$ & $5.5 \times 10^{-7}$ & 70,000 & & {$[3]$} \\
\hline
\end{tabular}

${ }^{a}$ Impurity concentrations are given in ppm unless otherwise stated. 
Thorium-Continued

\begin{tabular}{|c|c|c|c|c|c|c|c|c|c|c|}
\hline $\begin{array}{l}\text { Solvent } \\
\text { medium }\end{array}$ & $\begin{array}{l}\text { Diffusing } \\
\text { element }\end{array}$ & Purity ${ }^{a}$ & $\begin{array}{l}\text { Preparation and } \\
\text { properties }\end{array}$ & Method & $\begin{array}{l}\text { Diffusion } \\
\text { coefficient }\end{array}$ & $\begin{array}{c}\text { Temperature } \\
\text { range, }{ }^{\circ} \mathrm{C}\end{array}$ & $D_{0} \mathrm{~cm}^{2} / \mathrm{s}$ & $Q \mathrm{cal} / \mathrm{mol}$ & Comments & Ref. \\
\hline $\mathrm{ThO}_{2}$. & I... & Not stated. & $\begin{array}{l}\mathrm{ThO}_{2} \text { powder, was } \\
\text { irradiated for } 32 \\
\text { days at } 3 \times 10^{13} \\
\mathrm{n} / \mathrm{cm}^{2} / \mathrm{s} \text { (fast); } \\
\text { post-irradiation } \\
\text { anneals, I col- } \\
\text { lected on } \mathrm{Ag} \text { wool } \\
\text { at } 400{ }^{\circ} \mathrm{C} \text {. }\end{array}$ & $\begin{array}{l}\text { Measurement of } \\
\text { the rate of } \\
\text { release of } \\
\text { fission gas } \\
\text { from irradiated } \\
\text { powder. }\end{array}$ & $D_{T}$ & $800-1220$ & $7 \times 10^{-11}$ & 28,600 & & [4] \\
\hline $\mathrm{ThO}_{2} \ldots$ & Kr.......... & Not stated. & $\begin{array}{l}\text { Sintered } \mathrm{ThO}_{2} ; \mathrm{Kr} \\
\text { was introduced } \\
\text { by ion bombard. } \\
\text { ment. }\end{array}$ & $\begin{array}{l}\text { Measurement of } \\
\text { the rate of } \mathrm{Kr} \\
\text { release from } \\
\text { ion bombarded } \\
\text { sample. }\end{array}$ & $D_{T}$ & $1100-1350$ & & 104,000 & $\begin{array}{l}D_{0} \text { was assumed to } \\
\text { be } \sim 3 \times 10^{-1 \pm 1} \\
\mathrm{~cm}^{2} / \mathrm{s} \text { for } \\
\text { purposes of cal- } \\
\text { culating } D \text { from } \\
\text { the release data. }\end{array}$ & [5] \\
\hline $\mathrm{ThO}_{2} \ldots$ & $\mathrm{Kr} \ldots \ldots$. & $\begin{array}{c}\mathrm{Si} \sim 3 ; \mathrm{Mg} \text { and } \mathrm{Cu} \\
\sim 1 ; \text { all other } \\
\text { elements were } \\
\text { not detectable. }\end{array}$ & $\begin{array}{l}\mathrm{ThO}_{2} \text { powder; } \\
\text { irradiated with } \\
\text { fast neutron, } \\
\text { dosage } 10^{20} \text { nvt. }^{\mathrm{b}}\end{array}$ & $\begin{array}{l}\text { Measurement of } \\
\text { the rate of } \mathrm{Kr} \\
\text { release neutron } \\
\text { irradiated } \\
\text { powder. }\end{array}$ & $D_{T}$ & $600-1200$ & $2.5 \times 10^{-10}$ & 32,300 & & [6] \\
\hline $\mathrm{ThO}_{2}$. & $\mathrm{Nb} \ldots \ldots$ & Not stated. & $\begin{array}{l}\text { Grain size } 0.2 \mu \mathrm{m} ; \\
\text { irradiated with } \\
3.8 \times 10^{17} \text { fast } \\
\text { neutrons } / \mathrm{cm}^{2} .\end{array}$ & $\begin{array}{l}\text { Determination of } \\
\text { the rate of } \\
\text { leaching of } \\
\text { fission products } \\
\text { into molten } \mathrm{NaCl} \text {. }\end{array}$ & $D_{T}$ & $820-960$ & $8.3 \times 10^{-6}$ & 65,400 & & [3] \\
\hline $\mathrm{ThO}_{2} \ldots \ldots$ & O.......... & Not stated. & $\begin{array}{l}\text { Grain size about } \\
10 \mu \mathrm{m} .\end{array}$ & $\begin{array}{l}\text { Heterogeneous } \\
\text { isotopic exchange } \\
\text { between a solid } \\
\text { sample and a gas. }\end{array}$ & $\begin{array}{l}D^{*} \text { (self- } \\
\quad \text { diffusion) }\end{array}$ & $800-1420$ & $1 \times 10^{-8}$ & 14,700 & $\begin{array}{l}\text { Low values of } D_{0} \\
\text { and } Q \text { are sugges- } \\
\text { tive of grain- } \\
\text { boundary diffusion }\end{array}$ & [7] \\
\hline $\mathrm{ThO}_{2}$. & O.......... & $\begin{array}{l}\mathrm{Co}, \mathrm{Mg}, \mathrm{Fe} \text { each } \\
\quad<100 .\end{array}$ & $\begin{array}{l}\mathrm{ThO}_{2} \text { powder } \\
\text { fused into } \\
\text { spheres in a } \\
\text { plasma torch. }\end{array}$ & $\begin{array}{l}\text { Heterogeneous } \\
\text { isotopic ex- } \\
\text { change between } \\
\text { a solid sample } \\
\text { and a gas. }\end{array}$ & $D^{*}$ & $800-1500$ & 4.4 & 65,800 & $\begin{array}{l}\text { Finite rate of } \\
\text { exchange ac- } \\
\text { counted for in } \\
\text { treatment of } \\
\text { results. }\end{array}$ & [8] \\
\hline $\mathrm{ThO}_{2} \ldots$ & Rn...... & Not stated. & $\begin{array}{l}\mathrm{ThO}_{2} \text { was labeled } \\
\text { with } \mathrm{Rn} \text { by recoil } \\
\text { from } \alpha \text {-decay of } \\
\mathrm{Ra} \text { adsorbed on } \\
\text { the powder; the } \\
\mathrm{Ra} \text { was } \\
\text { quantitatively } \\
\text { removed after } \\
\text { labeling. }\end{array}$ & $\begin{array}{l}\text { Measurement of } \\
\text { the rate of } \\
\text { release of } \mathrm{Rn} \\
\text { from recoil } \\
\text { labeled powder. }\end{array}$ & $D_{T}$ & 565-1515 & & 59,000 & & [4] \\
\hline $\mathrm{ThO}_{2} \ldots$ & Rn... & Not stated. & $\begin{array}{l}\text { Sintered } \mathrm{ThO}_{2} ; \mathrm{Rn} \\
\text { was introduced } \\
\text { by recoil from } \alpha- \\
\text { decay of } \mathrm{Ra} \text { on } \\
\text { one face of sam- } \\
\text { ple; the Ra was } \\
\text { quantitatively re- } \\
\text { moved after } \\
\text { labeling. }\end{array}$ & $\begin{array}{l}\text { Measurement of } \\
\text { the rate of } \\
\text { release of } \mathrm{Rn} \\
\text { from recoil } \\
\text { labeled disk. }\end{array}$ & $D_{T}$ & $1100-1300$ & & 93,000 & $\begin{array}{l}D_{0} \text { was assumed } \\
\text { to be } \sim 3 \times 10^{-1 \pm 1} \\
\mathrm{~cm}^{2} / \mathrm{s} \text { for } \\
\text { purposes of cal- } \\
\text { culating } D \text { from } \\
\text { the release data. }\end{array}$ & [5] \\
\hline $\mathrm{ThO}_{2} .$. & Th.. & Not stated. & $\begin{array}{l}\mathrm{ThO}_{2} \text { pellets; } 97.5 \% \\
\text { of theoretical } \\
\text { density. }\end{array}$ & $\begin{array}{l}\text { Determination of } \\
\text { isotopic concen- } \\
\text { tration gradient } \\
\text { by mass spectro- } \\
\text { metric analysis } \\
\text { of thin sections. }\end{array}$ & $D^{*}$ & $925-1480$ & $4 \times 10^{-8}$ & 27,000 & $\begin{array}{l}\text { Data was adjusted } \\
\text { to eliminate grain- } \\
\text { boundary } \\
\text { contribution. }\end{array}$ & [9] \\
\hline $\mathrm{ThO}_{2}$. & Xe.. & Not stated. & $\begin{array}{l}\mathrm{ThO}_{2} \text { powder was } \\
\text { irradiated for } \\
32 \text { days at } 1.5 \\
\times 10^{14} \mathrm{n} / \mathrm{cm}^{2} \\
\text { (thermal), post- } \\
\text { irradiation } \\
\text { anneals. }\end{array}$ & $\begin{array}{l}\text { Measurement of } \\
\text { the rate of } \\
\text { release of } \\
\text { fission gas from } \\
\text { irradiated powder. }\end{array}$ & $D_{T}$ & $700-1220$ & & 30,000 & & [4] \\
\hline $\mathrm{ThO}_{2} \ldots$ & $\mathrm{Xe} \ldots$ & Not stated. & $\begin{array}{l}\text { Single crystal } \\
\text { ThO } \mathrm{O}_{2} ; \text { neutron } \\
\text { dose } \sim 3 \times 10^{16} \\
\text { n/cm } / \mathrm{cm}^{2} \text { (fast). }\end{array}$ & $\begin{array}{l}\text { Measurement of } \\
\text { the rate of } \\
\text { release of } \\
\text { fission gas from } \\
\text { irradiated } \\
\text { crystals. }\end{array}$ & $D_{T}$ & $1200-2000$ & $\begin{array}{c}1 \times 10^{-4} \\
-2 \times 10^{-2}\end{array}$ & 81,000 & $\begin{array}{c}\text { Based on prelimi- } \\
\text { nary } D / a^{2} \text { values } \\
\text { ( } a=\text { average } \\
\text { particle radius). }\end{array}$ & [10] \\
\hline $\mathrm{ThO}_{2} .$. & Xe..... & Not stated. & $\begin{array}{l}\text { Polycrystalline } \\
\mathrm{ThO}_{2} ; \text { neutron } \\
\text { dose } 7 \times 10^{15} \\
\text { n/cm }{ }^{2} \\
\text { (fast). }\end{array}$ & $\begin{array}{l}\text { Measurement of } \\
\text { the rate of } \\
\text { release of } \\
\text { fission gas } \\
\text { from irradiated } \\
\text { powder. }\end{array}$ & $D_{T}$ & $500-1100$ & & 37,000 & $\begin{array}{l}\text { Evaluated as } D / a^{2} \\
\text { values after sub- } \\
\text { traction of the } \\
\text { initial burst. }\end{array}$ & [10] \\
\hline $\mathrm{ThO}_{2} \ldots$ & Xe... & $\begin{array}{r}\text { Th, } 99.23 \mathrm{~m} / \mathrm{o} \\
\mathrm{U} 0.77 \mathrm{~m} / \mathrm{o}\end{array}$ & $\begin{array}{l}\mathrm{ThO}_{2} \text { powder; } \\
\text { neutron dose } \\
\sim 2.4 \times 10^{12} \mathrm{nvt} \\
\text { (thermal). }\end{array}$ & $\begin{array}{l}\text { Measurement of } \\
\text { the rate of } \\
\text { release of } \\
\text { fission gas } \\
\text { from irradiated } \\
\text { powder. }\end{array}$ & $D_{T}$ & $800-1600$ & & 50,000 & & [11] \\
\hline
\end{tabular}

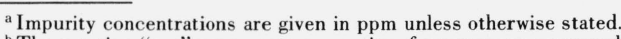

"The notation "nvt" represents a quantity of neutron exposure and has the units neutron- $\mathrm{cm}^{-2}$ 
Thorium-Continued

\begin{tabular}{|c|c|c|c|c|c|c|c|c|c|c|}
\hline $\begin{array}{l}\text { Solvent } \\
\text { medium }\end{array}$ & $\begin{array}{c}\text { Diffusing } \\
\text { element }\end{array}$ & Purity ${ }^{a}$ & $\begin{array}{l}\text { Preparation and } \\
\text { properties }\end{array}$ & Method & $\begin{array}{c}\text { Diffusion } \\
\text { coefficient }\end{array}$ & $\begin{array}{c}\text { Temperature } \\
\text { range, }{ }^{\circ} \mathrm{C}\end{array}$ & $D_{0} \mathrm{~cm}^{2} / \mathrm{s}$ & $Q \mathrm{cal} / \mathrm{mol}$ & Comments & Ref. \\
\hline $\mathrm{ThO}_{2} \ldots \ldots$ & Zr... & Not stated. & $\begin{array}{c}\text { Grain size } 0.2 \mu \\
\text { irradiated with } \\
3.8 \times 10^{17} \mathrm{fast} \\
\text { neutrons } / \mathrm{cm}^{2}\end{array}$ & $\begin{array}{l}\text { Determination of } \\
\text { the rate of leach- } \\
\text { ing of fission pro- } \\
\text { ducts into molten } \\
\mathrm{NaCl}\end{array}$ & $D_{T}$ & $840-960$ & $6.9 \times 10^{-7}$ & 62,300 & & [3] \\
\hline $\begin{array}{c}\mathrm{ThO}_{2}+ \\
12 \% \mathrm{UO}_{2}\end{array}$ & $\mathrm{Kr} \ldots \ldots \ldots$ & Not stated. & $\begin{array}{c}\text { Sintered } \mathrm{ThO}_{2}-\mathrm{UO}_{2} \\
\text { compact; } \mathrm{Kr} \text { was } \\
\text { introduced by ion } \\
\text { bombardment. }\end{array}$ & $\begin{array}{l}\text { Measurement of } \\
\text { the rate of } \mathrm{Kr} \\
\text { release from ion } \\
\text { bombarded } \\
\text { sample. }\end{array}$ & $D_{T}$ & $1250-1350$ & & 110,000 & $\begin{array}{l}\mathrm{D}_{0} \text { was assumed to } \\
\text { be } \sim 3 \times 10^{-1 \pm 1} \mathrm{~cm}^{2} / \\
\mathrm{s} \text { for purposes of } \\
\text { calculating } D \text { from } \\
\text { the release data. }\end{array}$ & [5] \\
\hline$\underset{10 \% \mathrm{Y}_{2} \mathrm{O}_{3}}{\mathrm{ThO}_{2}+}$ & Se .......... & $\begin{array}{l}\text { Th } 89.39 \mathrm{~m} / \mathrm{o} \\
\text { U, } 0.70 \mathrm{~m} / \mathrm{o} ; \mathrm{Y}, \\
9.91 \mathrm{~m} / \mathrm{o} .\end{array}$ & $\begin{array}{l}\text { Neutron dose } \sim 2.4 \\
\times 10^{17} \mathrm{nvt}(\text { thermal })\end{array}$ & $\begin{array}{l}\text { Measurement of } \\
\text { the rate of } \\
\text { release of } \\
\text { fission gas } \\
\text { from irradiat- } \\
\text { ed powder. }\end{array}$ & $D_{T}$ & $1000-1600$ & & 75,000 & & [11] \\
\hline $\begin{array}{l}\mathrm{ThO}_{2}+ \\
30 \% \mathrm{Y}_{2} \mathrm{O}_{3}\end{array}$ & Se ........... & $\begin{array}{l}\text { Th } 69.48 \mathrm{~m} / \mathrm{o} ; \mathrm{U} \\
0.74 \mathrm{~m} / \mathrm{o} ; \mathrm{Y} \\
29.78 \mathrm{~m} / \mathrm{o}\end{array}$ & $\begin{array}{l}\text { Neutron dose } \sim 2.4 \\
\quad \times 10^{17} \mathrm{nvt}(\text { thermal) }\end{array}$ & $\begin{array}{l}\text { Measurement of } \\
\text { the rate of } \\
\text { release of } \\
\text { fission gas } \\
\text { from irradiat. } \\
\text { ed powder. }\end{array}$ & $D_{T}$ & $900-1600$ & & 90,000 & & [11] \\
\hline
\end{tabular}

ampurity concentrations are given in ppm unless otherwise stated.

b The notation "nvt" represents a quantity of neutron exposure and has the units neutron- $\mathrm{cm}^{-2}$.

[1] D. T. Peterson, Trans. ASM 53, 765 (1961).

[2] J. F. Smith and G. C. Danielson, J. Chem. Phys. 22, 266 (1954).

[3] R. Thomas and R. Lindner, Radiochimica Acta 4, 170 (1965).

[4] Hj. Matzke and R. Lindner, Z. Naturforsch 15a, 647 (1960).

[5] R. Kelly and Hj. Matzke, J. Nuclear Mater. 17, 179 (1965).

[6] Hj. Matzke, Z Naturforsch. 16a, 1255 (1961)

[7] C. S. Morgan and C. S. Yust, U.'S. At. Energy Comm. Report ORNL-3160, 41 (1961).

[8] H. S. Edwards, A. F. Rosenberg, and J. T. Bittel, Technical Documentary Report ASD-TDR-63-635, 2 (1963),

[9] C. S. Morgan and C. S. Yust, U. S. At. Energy Comm. Report ORNL-3313, 142 (1962).

[10]F. Felix, T. Lagerwall, P. Schmeling, and K. E. Zimen, Third Geneva Conf. A/CONF. 28/P/472, 363 (1964).

[11] G. Long, W. P. Stanaway, and D. Davies, At. Energy Research Establishment Report AERE-M 1251 (1964).

Titanium

\begin{tabular}{|c|c|c|c|c|c|c|c|c|c|c|}
\hline $\begin{array}{l}\text { Solvent } \\
\text { medium }\end{array}$ & $\begin{array}{l}\text { Diffusing } \\
\text { element }\end{array}$ & Purity ${ }^{a}$ & $\begin{array}{l}\text { Preparation and } \\
\text { properties }\end{array}$ & Method & $\begin{array}{c}\text { Diffusion } \\
\text { coefficient }\end{array}$ & $\begin{array}{c}\text { Temperature } \\
\text { range, }{ }^{\circ} \mathrm{C}\end{array}$ & $D_{0} \mathrm{~cm}^{2} / \mathrm{s}$ & $Q \mathrm{cal} / \mathrm{mol}$ & Comments & Ref. \\
\hline$\alpha$-Ti.. & C............ & $\begin{array}{l}\text { Other than C: } \mathrm{O}, \\
\text { 180-2360 (highest } \\
\text { in C-Ti alloy); } \\
\mathrm{H}, 90-200 ; \mathrm{N}, \\
110-340 ; \mathrm{Al}, 30 ; \\
\mathrm{Fe}, 10-70 ; \mathrm{Si}, \\
20-50 ; \mathrm{Mn}, 10-30 ; \\
\text { all others } \leqq 10 \text {. }\end{array}$ & $\begin{array}{l}\text { C-Ti alloy }(0.4 \mathrm{w} / \mathrm{o} \\
\text { or higher in } \mathrm{C}) \\
\text { buttwelded to high } \\
\text { purity Ti }(0.038 \\
\text { w/o C). }\end{array}$ & $\begin{array}{l}\text { Determination of } \\
\text { the concentration } \\
\text { gradient by chemi- } \\
\text { cal analysis. }\end{array}$ & $\begin{array}{l}\tilde{D} \text { (chemical } \\
\text { diffusion } \\
\text { coefficient). }\end{array}$ & $736-882$ & 5.06 & 43,500 & $\begin{array}{l}\bar{D} \text { was assumed to } \\
\text { be independent of } \\
\mathrm{C} \text { concentration. }\end{array}$ & [1] \\
\hline$\beta$-Ti.... & C............. & & $\begin{array}{l}\text { C-Ti alloy }(0.4 \mathrm{w} / \mathrm{o} \\
\text { or higher in } \mathrm{C}) \\
\text { buttwelded to high } \\
\text { purity Ti }(0.038 \\
\text { w/o C). }\end{array}$ & $\begin{array}{l}\text { Determination of } \\
\text { the concentration } \\
\text { gradient by chemi- } \\
\text { cal analysis. }\end{array}$ & $D$ & $920-1150$ & 108 & 48,400 & $\begin{array}{l}\tilde{D} \text { was assumed to } \\
\text { be independent of } \\
\text { concentration. }\end{array}$ & [1] \\
\hline$\beta-\mathrm{Ti} \ldots$. & N........ & $\begin{array}{l}\text { Principal impur- } \\
\text { ities: } \mathrm{C}, 360 ; \\
\mathrm{Mn}, 300 ; \mathrm{O}, 110 ; \\
\mathrm{Al}, 100 . \\
\text { Commercial } \mathrm{N}_{2} \\
\text { passed through } \\
\text { liquid } \mathrm{N}_{2} \text { trap or } \\
\text { tube furnace with } \\
\text { Ti turnings. }\end{array}$ & $\begin{array}{l}\text { Argon-arc-melted } \\
\text { iodide Ti, hot- } \\
\text { forged and rolled, } \\
\text { swaged cold to } \\
\text { rod-shaped, ma- } \\
\text { chined into cy- } \\
\text { lindrical } \\
\text { specimens. }\end{array}$ & $\begin{array}{l}\text { Determination of } \\
\text { the concentration } \\
\text { gradient by chemi- } \\
\text { cal analysis } \\
\text { (Kjeldahl). }\end{array}$ & $\tilde{D}$ & $800-1400$ & $3.5 \times 10^{-2}$ & 33,800 & $\begin{array}{l}\text { Measurements were } \\
\text { complicated by } \\
\text { formation of } \\
\text { outer nitride } \\
\text { layer and } \alpha- \\
\text { phase, but less } \\
\text { than } 3 \% \text { of } \\
\text { cylindrical radius; } \\
\tilde{D} \text { was assumed to } \\
\text { be independent of } \\
\text { concentration. }\end{array}$ & [2] \\
\hline$\alpha-\mathrm{Ti} \ldots$ & $0 \ldots$ & Not stated. & $\begin{array}{l}\text { VT-1 Ti, samples } \\
\text { were polished and } \\
\text { then etched for } \\
60 \mathrm{~s} \text { in } 7 \% \mathrm{Hf} \\
\text { in } \mathrm{HNO}_{3} .\end{array}$ & $\begin{array}{l}\text { Determination of } \\
\text { the concentra- } \\
\text { tion gradient by } \\
\text { microhardness } \\
\text { measurements. }\end{array}$ & $\tilde{\tilde{D}}$ & $800-900$ & $1.80 \times 10^{-3}$ & 35,900 & $\begin{array}{l}\tilde{D} \text { was assumed to } \\
\text { be independent of } \\
\text { concentration; } \\
\text { hardness was } \\
\text { assumed to be } \\
\text { linearly depend- } \\
\text { ent on the concen- } \\
\text { tration of } O \text {. }\end{array}$ & [3] \\
\hline$\alpha-\mathrm{Ti}_{\ldots} \ldots$ & $0 \ldots$ & $\begin{array}{l}\mathrm{Fe}, \mathrm{Mn}, \mathrm{Al}, \mathrm{Sn}, \\
\mathrm{Mg} \text { and } \mathrm{Cr} \text {, each } \\
100 ; \mathrm{Cu}, 10 .\end{array}$ & $\begin{array}{l}\text { Electron-beam- } \\
\text { melted titanium. }\end{array}$ & $\begin{array}{l}\text { Determination of } \\
\text { the concentration } \\
\text { gradient by } \\
\text { microhardness } \\
\text { measurements. }\end{array}$ & $\tilde{D}$ & $800-1000$ & 0.5 & 51,000 & $\begin{array}{l}\tilde{D} \text { was assumed to } \\
\text { be independent of } \\
\text { concentration; } \\
\text { hardness was as- } \\
\text { sumed to be lin- } \\
\text { early dependent } \\
\text { on concentration } \\
\text { of } 0 .\end{array}$ & [4] \\
\hline
\end{tabular}

${ }^{a}$ Impurity concentrations are given in ppm unless otherwise stated. 
Titanium-Continued

\begin{tabular}{|c|c|c|c|c|c|c|c|c|c|c|}
\hline $\begin{array}{l}\text { Solvent } \\
\text { medium }\end{array}$ & $\begin{array}{l}\text { Diffusing } \\
\text { element }\end{array}$ & Purity ${ }^{a}$ & $\begin{array}{l}\text { Preparation and } \\
\text { properties }\end{array}$ & Method & $\begin{array}{l}\text { Diffusion } \\
\text { coefficient }\end{array}$ & $\begin{array}{c}\text { Temperature } \\
\text { range, }{ }^{\circ} \mathrm{C}\end{array}$ & $D_{0} \mathrm{~cm}^{2} / \mathrm{s}$ & $Q \mathrm{cal} / \mathrm{mol}$ & Comments & Ref. \\
\hline$\alpha-\mathrm{Ti}_{\ldots} .$. & $\mathrm{O} \ldots \ldots$ & $\begin{array}{l}\text { Si, } 600 ; \mathrm{Fe}, \\
1500 ; \mathrm{Al}, 2000 ; \\
\mathrm{Mg}, 200 ; \mathrm{Mn}, \\
100-500 ; \mathrm{W}, 300 ; \\
\text { N, } 100 ; \text { all } \\
\text { others } \leqq 100 .\end{array}$ & $\begin{array}{l}\text { Ti bars were } \\
\text { oxidized by } \mathrm{TiO}_{2} \\
\text { powder. }\end{array}$ & $\begin{array}{l}\text { Determination of } \\
\text { the concentra- } \\
\text { tion gradient by } \\
\text { microhardness } \\
\text { measurements. }\end{array}$ & $\widetilde{D}$ & $700-850$ & $5.08 \times 10^{-3}$ & 33,500 & $\begin{array}{l}\widetilde{D} \text { was assumed to } \\
\text { be independent of } \\
\text { concentration; } \\
\text { hardness was } \\
\text { assumed to be } \\
\text { linearly depend- } \\
\text { ent on the con- } \\
\text { centration of } \mathrm{O} \text {. }\end{array}$ & [5] \\
\hline$\beta-\mathrm{Ti} \ldots$ & $0 \ldots$ & $\begin{array}{l}\text { Principal impur- } \\
\text { ities: } \mathrm{C}, 360 ; \\
\mathrm{Mn}, 300 ; \mathrm{O}, 110 ; \\
\text { Al, } 100 \text {. Dried } \\
\text { commercial } \mathrm{O}_{2} \\
\text { and } \mathrm{O}_{2} \text { from } \\
\text { decomposition of } \\
\text { permanganate } \\
\text { were used. }\end{array}$ & $\begin{array}{l}\text { Argon-arc-melted } \\
\text { iodide Ti, hot- } \\
\text { forged and rolled, } \\
\text { swaged cold to } \\
\text { rod-shape, ma- } \\
\text { chined into } \\
\text { cylindrical } \\
\text { specimens. }\end{array}$ & $\begin{array}{l}\text { Determination of } \\
\text { the concentration } \\
\text { gradient by chem- } \\
\text { ical analysis } \\
\text { (vacuum fusion } \\
\text { with tin). }\end{array}$ & $\tilde{D}$ & $950-1414$ & 1.6 & 48,200 & $\begin{array}{l}\widetilde{D} \text { was assumed to } \\
\text { be independent of } \\
\text { concentration. }\end{array}$ & [2] \\
\hline$\beta-\mathrm{Ti} \ldots \ldots$ & $0 \ldots \ldots$ & $\begin{array}{l}\mathrm{Si}, 600 ; \mathrm{Fe}, \\
\quad 1500 ; \mathrm{Al}, 2000 ; \\
\mathrm{Mg}, 200 ; \mathrm{Mn}, 10- \\
50 ; \mathrm{W}, 300 ; \mathrm{Ni}, \\
10 ; \text { all others } \\
\leqq 100 .\end{array}$ & $\begin{array}{l}\text { Ti cylinders were } \\
\text { oxidized by } \mathrm{TiO}_{2} \\
\text { powder. }\end{array}$ & $\begin{array}{l}\text { Determination of } \\
\text { the concentra- } \\
\text { tion gradient by } \\
\text { microhardness } \\
\text { measurements. }\end{array}$ & $\tilde{D}$ & $930-1150$ & $3.14 \times 10^{4}$ & 68,700 & $\begin{array}{l}\text { Measurements were } \\
\text { complicated by } \\
\text { grain growth and } \\
\text { formation of the } \\
\alpha \text {-phase. } \widetilde{D} \text { was } \\
\text { assumed to be } \\
\text { independent of } \\
\text { concentration; } \\
\text { hardness was } \\
\text { assumed to be } \\
\text { linearly dependent } \\
\text { on concentration. }\end{array}$ & [5] \\
\hline$\beta$-Ti...... & $0 \ldots \ldots \ldots$ & $\begin{array}{l}\text { Ti, } 99.7 \text { w/o, } \\
\text { Cu major im- } \\
\text { purity. }\end{array}$ & $\begin{array}{l}\text { Ti-wire }(\mathrm{Ti}-75 \mathrm{~A}) ; \\
\text { initially } \sim 1 \mathrm{~mm} \\
\text { section of wire } \\
\text { oxidized in } \mathrm{O}_{2} .\end{array}$ & $\begin{array}{l}\text { Determination of } \\
\text { the concentra- } \\
\text { tion gradient by } \\
\text { change in resist- } \\
\text { ance along the } \\
\text { sample. }\end{array}$ & $\tilde{D}$ & $1130-1350$ & 0.083 & 31,200 & $\begin{array}{l}\text { Results may be } \\
\text { complicated by } \\
\text { formation of } \alpha \text { - } \\
\text { phase which would } \\
\text { be stabilized by } \\
\text { oxygen atoms in } \\
\text { the metal; linear } \\
\text { dependence of re- } \\
\text { sistance on } O \\
\text { concentration up } \\
1 \% O \text { was verified } \\
\text { by [24]. }\end{array}$ & [6] \\
\hline $\mathrm{TiB}_{2}$. & B... & Not stated. & $\begin{array}{l}\text { Boronization of } \\
\text { surface of } \mathrm{Ti} \text { - } \\
\text { cylinder by heat- } \\
\text { ing in a boron } \\
\text { carbide-borax } \\
\text { charge. }\end{array}$ & $\begin{array}{l}\text { Determination of } \\
\text { the concentra- } \\
\text { tion gradient by } \\
\text { microhardness } \\
\text { measurements. }\end{array}$ & $\widetilde{D}$ & $1100-1400$ & $8.9 \times 10^{-5}$ & 30,600 & $\begin{array}{l}\tilde{D} \text { was assumed to } \\
\text { be independent of } \\
\text { B concentration. }\end{array}$ & [7] \\
\hline TiC.. & C.... & Not stated. & $\begin{array}{l}\text { Carburization of } \\
\text { the surface of } \mathrm{Ti} \\
\text { cylinder by heat- } \\
\text { ing in a boron } \\
\text { carbide-borax } \\
\text { charge. }\end{array}$ & $\begin{array}{l}\text { Determination of } \\
\text { the concentration } \\
\text { gradient by } \\
\text { microhardness } \\
\text { measurements. }\end{array}$ & $\tilde{D}$ & $900-1300$ & $2.04 \times 10^{-3}$ & 33,000 & $\begin{array}{l}\tilde{D} \text { was assumed to be } \\
\text { independent of } \\
\text { concentration. }\end{array}$ & [7] \\
\hline $\mathrm{TiC} \ldots . .$. & $\mathrm{Nb} . . . \ldots$. & $\begin{array}{l}\text { Free C, } 4000 ; \\
\quad \text { Fe, } 150 ; \mathrm{N}, 350 .\end{array}$ & $\begin{array}{l}\text { Carburization of } \\
\mathrm{TiO}_{2}+\mathrm{C} \text { in vac- } \\
\text { uum furnace with } \\
\text { graphite heater, } \\
\text { carbide was } \\
\text { crushed, } \mathrm{Fe} \text { was } \\
\text { removed with } \\
\mathrm{HCl} \text {; density ap- } \\
\text { proximated theo- } \\
\text { retical values. }\end{array}$ & $\begin{array}{l}\text { Method of Zhuk- } \\
\text { hovitskii and } \\
\text { Geodakyan [8]; } \\
\text { nonexponential } \\
\text { attenuation of } \\
\beta \text {-radiation } \\
\text { when a radio- } \\
\text { isotope diffuses } \\
\text { into a solid. }\end{array}$ & $\begin{array}{l}D_{T} \text { (tracer } \\
\quad \text { diffusion). }\end{array}$ & $1935-2170$ & 2.4 & 84,000 & & [9] \\
\hline $\begin{array}{c}\mathrm{TiC} \cdot \mathrm{NbC} . . \\
(1: 1)\end{array}$ & $\mathrm{Nb} . . . \ldots . .$. & $\begin{array}{l}\text { Free C, } 2600 ; \text { Fe. } \\
200 ; \text { N, } 700 .\end{array}$ & $\begin{array}{l}\text { Carburization of } \\
\mathrm{TiO}_{2}+\mathrm{NbC}+\mathrm{C} \\
\text { in vacuum fur- } \\
\text { nace, etc. }\end{array}$ & $\begin{array}{l}\text { Method of } \\
\text { Zhukhovitskii } \\
\text { and Geodakyan. }\end{array}$ & $\begin{array}{l}D^{*} \text { (self- } \\
\text { diffusion). }\end{array}$ & $2170-2280$ & 470 & 120,000 & & [9] \\
\hline $\mathrm{TiC} \ldots \ldots \ldots . .$. & W........... & Free C, 1100 & $\begin{array}{l}\text { Carburization of } \\
\mathrm{TiO}_{2} \text { in vacuum } \\
\text { furnace; porosity } \\
\text { up to } 0.2 \% \text {; } \\
\text { grain size } \\
50-100 \mu \mathrm{m} \text {; den- } \\
\text { sity } 4.97 \mathrm{~g} / \mathrm{cm}^{3} \text {. }\end{array}$ & $\begin{array}{l}\text { Method of Kryukov } \\
\text { and Zhukhovitskii } \\
\text { [10]; diffusion of } \\
\text { thin radioactive } \\
\text { layer into thin } \\
\text { sample }(30 \text { to } \\
100 \mu \mathrm{m}) \text {. }\end{array}$ & $D_{T}$ & $2130-2300$ & 7.7 & 115,000 & $\begin{array}{l}\text { There may be some } \\
\text { error in this data } \\
\text { due to vaporiza- } \\
\text { tion of the radio- } \\
\text { active layer. }\end{array}$ & [11] \\
\hline $\begin{array}{l}\text { TiC } \cdot \text { WC... } \\
\quad(77: 23)\end{array}$ & W.......... & Free C, 1700. & $\begin{array}{l}\text { Carburization in } \\
\text { vacuum furnace; } \\
\text { porosity } 0.2 \% ; \\
\text { grain size } 50-70 \\
\mu \mathrm{m} ; \text { density } 7.35 \\
\mathrm{~g} / \mathrm{cm}^{3} \text {. }\end{array}$ & $\begin{array}{l}\text { Method of Kryukov } \\
\text { and Zhukhovitskii. }\end{array}$ & $D^{*}$ & $2130-2300$ & 31.6 & 138,000 & $\begin{array}{l}\text { Measurements also } \\
\text { were made on } \\
\text { other mixed car- } \\
\text { bides, but these } \\
\text { appear to have } \\
\text { large errors due to } \\
\text { vaporization of } \\
\text { the radioactive } \\
\text { layer. }\end{array}$ & [11] \\
\hline
\end{tabular}

${ }^{\mathrm{a}}$ Impurity concentrations are given in ppm unless otherwise stated. 
Titanium-Continued

\begin{tabular}{|c|c|c|c|c|c|c|c|c|c|c|}
\hline $\begin{array}{l}\text { Solvent } \\
\text { medium }\end{array}$ & $\begin{array}{l}\text { Diffusing } \\
\text { element }\end{array}$ & Purity ${ }^{a}$ & $\begin{array}{l}\text { Preparation and } \\
\text { properties }\end{array}$ & Method & $\begin{array}{l}\text { Diffusion } \\
\text { coefficient }\end{array}$ & $\begin{array}{c}\text { Temperature } \\
\text { range, }{ }^{\circ} \mathrm{C}\end{array}$ & $D_{0} \mathrm{~cm}^{2} / \mathrm{s}$ & $Q \mathrm{cal} / \mathrm{mol}$ & Comments & Ref. \\
\hline $\mathrm{TiO}_{2} \ldots \ldots$ & Ba........ & $\begin{array}{l}\text { Rutile: traces of } \\
\text { Si and } \mathrm{Fe} \\
\mathrm{Ba}: \mathrm{Ca}, 2 ; \mathrm{Mg}, 1 ; \\
\mathrm{Cu}, 1 ; \mathrm{Na}, 1 .\end{array}$ & $\begin{array}{l}{ }^{133} \mathrm{Ba} \text { labeled car- } \\
\text { bonate was de- } \\
\text { composed to } \\
\text { oxide on } \mathrm{TiO}_{2} \\
\text { single crystal in } \\
\mathrm{O}_{2} \text { atmosphere, } \\
\text { second-phase } \\
\text { region formed in } \\
\mathrm{TiO}_{2} \text {. }\end{array}$ & $\begin{array}{l}\text { Determination of } \\
\text { the concentration } \\
\text { gradient by radio- } \\
\text { isotope distribu- } \\
\text { tion, Matano } \\
\text { analysis }[12,13] \text {. }\end{array}$ & $\begin{array}{l}\bar{D} \text {, perpen- } \\
\text { dicular to } C \\
\text { axis of } \\
\text { crystal. }\end{array}$ & $1100-1200$ & & $\sim 59,000$ & & [14] \\
\hline $\mathrm{TiO}_{2}, \ldots \ldots \ldots$ & Ba........ & $\begin{array}{l}\text { Rutile: traces of } \\
\text { Si and } \mathrm{Fe} \\
\mathrm{Ba}: \mathrm{Ca}, 2 ; \mathrm{Mg}, 1 ; \\
\mathrm{Cu}, 1 ; \mathrm{Na}, 1 .\end{array}$ & $\begin{array}{l}{ }^{133} \mathrm{Ba} \text { labeled carbo- } \\
\text { nate was decom- } \\
\text { posed to oxide on } \\
\mathrm{TiO}_{2} \text { single crys- } \\
\text { tal in } \mathrm{O}_{2} \text { atmos- } \\
\text { phere, second- } \\
\text { phase region } \\
\text { formed in } \mathrm{TiO}_{2} .\end{array}$ & $\begin{array}{l}\text { Determination of } \\
\text { the concentration } \\
\text { gradient by radio- } \\
\text { isotope distribu- } \\
\text { tion, Matano } \\
\text { analysis. }\end{array}$ & $\begin{array}{l}\tilde{D} \text {, parallel to } \\
C \text { axis of } \\
\text { crystal. }\end{array}$ & $1100-1200$ & & $\sim 43,000$ & & [14] \\
\hline $\mathrm{TiO}_{2} \ldots \ldots$ & Fe......... & $\begin{array}{l}\text { Impurities not } \\
\text { precipitated } \\
\text { with } \mathrm{NH}_{3}, 1000 ; \\
\mathrm{Fe}, 100 ; \text { heavy } \\
\text { metals of sulfide } \\
\text { type, } 500 \text {. }\end{array}$ & $\begin{array}{l}\mathrm{TiO}_{2} \text { powder was } \\
\text { compressed into } \\
\text { pellets and } \\
\text { sintered at } 1100 \\
{ }^{\circ} \mathrm{C} ;{ }^{59} \mathrm{Fe} \text { was } \\
\text { vacuum deposited } \\
\text { on pellet face. }\end{array}$ & $\begin{array}{l}\text { Determination of } \\
\text { the concentra- } \\
\text { tion gradient by } \\
\text { radioisotope } \\
\text { distribution. }\end{array}$ & $D_{T}$ (air) & $800-1000$ & $1.98 \times 10^{-2}$ & 55,000 & & [15] \\
\hline $\mathrm{TiO}_{2} \ldots \ldots$ & $\mathrm{Fe} . .$. & $\begin{array}{l}\text { Impurities not } \\
\text { precipitated } \\
\text { with } \mathrm{NH}_{3}, 1000 ; \\
\mathrm{Fe}, 100 ; \text { heavy } \\
\text { metals of sulfide } \\
\text { type, } 500 \text {. }\end{array}$ & $\begin{array}{l}\mathrm{TiO}_{2} \text { powder was } \\
\text { compressed into } \\
\text { pellets and } \\
\text { sintered at } 1100 \\
{ }^{\circ} \mathrm{C} ;{ }^{59} \mathrm{Fe} \text { was } \\
\text { vacuum deposited } \\
\text { on pellet face. }\end{array}$ & $\begin{array}{l}\text { Determination of } \\
\text { the concentra- } \\
\text { tion gradient bv } \\
\text { radioisotope } \\
\text { distribution. }\end{array}$ & $D_{T}$ (vacuum). & $770-1000$ & 0.192 & 55,400 & & [15] \\
\hline $\mathrm{TiO}_{2 .}$. & $\mathrm{Fe} \ldots \ldots \ldots$ & $\begin{array}{l}\text { Impurities not } \\
\text { precipitated } \\
\text { with } \mathrm{NH}_{3}, 1000 ; \\
\mathrm{Fe}, 100 ; \text { heavy } \\
\text { metals of sulfide } \\
\text { type, } 500 \text {. }\end{array}$ & $\begin{array}{l}\mathrm{TiO}_{2} \text { powder was } \\
\text { compressed into } \\
\text { pellets and } \\
\text { sintered at } 1100 \\
{ }^{\circ} \mathrm{C} ;{ }^{59} \mathrm{Fe} \text { was vac- } \\
\text { uum deposited } \\
\text { on pellet face. }\end{array}$ & $\begin{array}{l}\text { Determination of } \\
\text { the concentra- } \\
\text { tion gradient by } \\
\text { radioisotope } \\
\text { distribution. }\end{array}$ & $\begin{array}{l}D_{\mathrm{gr}} \text {, grain- } \\
\text { boundary dif- } \\
\text { fusion in the } \\
\text { presence of air. }\end{array}$ & $800-1000$ & $1.56 \times 10^{-5}$ & 34,000 & & [15] \\
\hline $\mathrm{TiO}_{2 . .}$ & Fe... & $\begin{array}{l}\text { Impurities not } \\
\text { precipitated } \\
\text { with } \mathrm{NH}_{3}, 1000 ; \\
\mathrm{Fe}, 100 ; \text { heavy } \\
\text { metals of sulfide } \\
\text { type, } 500 \text {. }\end{array}$ & $\begin{array}{l}\mathrm{TiO}_{2} \text { powder was } \\
\text { compressed into } \\
\text { pellets and sin- } \\
\text { tered at } 1100^{\circ} \mathrm{C} ; \\
{ }^{59} \mathrm{Fe} \text { was vacuum } \\
\text { deposited on } \\
\text { pellet face. }\end{array}$ & $\begin{array}{l}\text { Determination of } \\
\text { the concentra- } \\
\text { tion gradient by } \\
\text { radioisotope } \\
\text { distribution. }\end{array}$ & $D_{\mathrm{gr}}$ (vacuum) & $770-1000$ & $1.13 \times 10^{-3}$ & 34,700 & & [15] \\
\hline $\mathrm{TiO}_{2} \ldots \ldots$ & Li........... & Not stated. & $\begin{array}{l}\text { Single crystal } \mathrm{TiO}_{2} \\
\text { doped uniformly } \\
\text { with Li. }\end{array}$ & $\begin{array}{l}\text { Measurement of } \\
\text { the change in } \\
\text { optical absorp- } \\
\text { tion due to the } \\
\text { diffusion of } \mathrm{Li} \text { out } \\
\text { of the crystal. }\end{array}$ & $\begin{array}{l}\tilde{D} \text {, parallel to } \\
C \text { axis of } \\
\text { crystal. }\end{array}$ & $80-550$ & 0.295 & 7,610 & $\begin{array}{l}\text { Impurity concentra- } \\
\text { tion and surface } \\
\text { damage were ob- } \\
\text { served to affect } \\
\text { diffusion rates; } \\
\text { impurities were } \\
\text { minimized by } \\
\text { taking cuts near } \\
\text { center of boule; } \\
\text { abrasion of sur- } \\
\text { faces was used to } \\
\text { control diffusion } \\
\text { direction. }\end{array}$ & [16] \\
\hline $\mathrm{TiO}_{2} \ldots \ldots$ & $0 \ldots \ldots$ & $\begin{array}{l}\mathrm{Al}_{2} \mathrm{O}_{3}, 100 \text { to } \\
200 ; \text { infrared } \\
\text { measurements } \\
\text { showed that } \mathrm{OH} \text { - } \\
\text { groups were } \\
\text { present. }\end{array}$ & $\begin{array}{l}\text { Single crystal } \mathrm{TiO}_{2} \\
\text { pulverized to } 10- \\
250 \mu \mathrm{m} .\end{array}$ & $\begin{array}{l}\text { Heterogeneous } \\
\text { isotopic ex- } \\
\text { change between } \\
\text { solid sample and } \\
\text { a gas. }\end{array}$ & $\begin{array}{l}D^{*} \text { (self- } \\
\text { diffusion). }\end{array}$ & $700-1300$ & $2.0 \times 10^{-3}$ & 60,000 & $\begin{array}{l}\text { "High" } \mathrm{Al}_{2} \mathrm{O}_{3} \text { con- } \\
\text { centration may } \\
\text { have caused } \\
\text { extrinsic diffusion. }\end{array}$ & {$[17,18]$} \\
\hline $\mathrm{TiO}_{2}, \ldots \ldots \ldots$ & Rn ............. & Not stated. & $\begin{array}{c}\mathrm{TiO}_{2} \text { powder labeled } \\
\text { with } \mathrm{Rn} \text { by recoil } \\
\text { from } \mathrm{Ra} \text { decay. }\end{array}$ & $\begin{array}{l}\text { Hahn emanation } \\
\text { technique, } \\
\text { modified by } \\
\text { mixing oxide } \\
\text { powder with Ra } \\
\text { salt allowing } \mathrm{Rn} \\
\text { to recoil into } \\
\text { oxide and then } \\
\text { dissolving away } \\
\text { the Ra. }\end{array}$ & $D_{T}$ & $315-1150$ & & 22,000 & & [19] \\
\hline $\mathrm{TiO}_{2} \ldots \ldots$ & Sn.............. & Not stated. & $\begin{array}{l}\mathrm{TiO}_{2} \text { single crystal; } \\
\mathrm{SnO}_{2} \text { applied to } \\
\text { face of crystal. }\end{array}$ & $\begin{array}{l}\text { Determination of } \\
\text { the concentra- } \\
\text { tion gradient by } \\
\text { x-ray analysis } \\
\text { (change in the } \\
\text { intensity of } \mathrm{x} \\
\text { rays reflected } \\
\text { as TiK } \text { TiKd }_{\alpha} \text { and } \\
\mathrm{SnL}_{\alpha 1} \text {. }\end{array}$ & $\begin{array}{l}D_{T}, \text { parallel } \\
\text { to } C \text { axis } \\
\text { of crystal. }\end{array}$ & $900-1050$ & 0.16 & 80,000 & & [20] \\
\hline
\end{tabular}

${ }^{\mathrm{a}}$ Impurity concentrations are given in ppm unless otherwise stated. 
Titanium-Continued

\begin{tabular}{|c|c|c|c|c|c|c|c|c|c|c|}
\hline $\begin{array}{l}\text { Solvent } \\
\text { medium }\end{array}$ & $\begin{array}{c}\text { Diffusing } \\
\text { element }\end{array}$ & Purity ${ }^{a}$ & $\begin{array}{l}\text { Preparation and } \\
\text { properties }\end{array}$ & Method & $\begin{array}{l}\text { Diffusion } \\
\text { coefficient }\end{array}$ & $\begin{array}{c}\text { Temperature } \\
\text { range, }{ }^{\circ} \mathrm{C}\end{array}$ & $D_{0} \mathrm{~cm}^{2} / \mathrm{s}$ & $Q \mathrm{cal} / \mathrm{mol}$ & Comments & Ref. \\
\hline $\mathrm{BaTiO}_{3} \ldots \ldots$ & Ba....... & Not stated. & $\begin{array}{l}\mathrm{BaCO}_{3} \text { reacted } \\
\text { with } \mathrm{TiO}_{2} \text { to form } \\
\mathrm{BaTiO}_{3} ; \text { com- } \\
\text { pressed and sin- } \\
\text { tered, } 80 \% \text { of the- } \\
\text { oretical density. }\end{array}$ & $\begin{array}{l}\text { Determination of } \\
\text { the concentration } \\
\text { gradient by radio- } \\
\text { isotope distribu- } \\
\text { tion. }\end{array}$ & $D^{*}$ & $884-1180$ & 0.8 & 89,000 & & [21] \\
\hline $\mathrm{SrTiO}_{3} \ldots \ldots$ & O...... & $\begin{array}{l}\mathrm{Mg} \leqq 1 ; \mathrm{Si} \sim 100 \\
\mathrm{Al} \leqq 10 ; \mathrm{Au} \leqq 1 \\
\mathrm{~Pb}, 10\end{array}$ & $\begin{array}{l}\text { Single crystal; } \\
\quad \text { dislocation } \\
\text { density of } \sim 1.4 \\
\times 10^{6} / \mathrm{cm}^{2} .\end{array}$ & $\begin{array}{l}\text { Heterogeneous } \\
\text { isotopic ex- } \\
\text { change between } \\
\text { a solid sample } \\
\text { and a gas. }\end{array}$ & $D^{*}$ & $825-1200$ & $1.6 \times 10^{-7}$ & 15,500 & $\begin{array}{l}\text { Low frequency } \\
\text { factor, } D_{0} \text {, was } \\
\text { taken as evidence } \\
\text { for extrinsic diffu- } \\
\text { sion; diffusion rate } \\
\text { was found to de- } \\
\text { pend on disloca- } \\
\text { tion density. }\end{array}$ & [22] \\
\hline $\mathrm{SrTiO}_{3} \ldots \ldots$ & $0 \ldots \ldots$ & $\begin{array}{l}\mathrm{Mg} \leqq ; \mathrm{Si} \sim 10 \\
\mathrm{Al} \leqq 10 ; \mathrm{Au} \leqq 1 \\
\mathrm{~Pb}, 10\end{array}$ & $\begin{array}{l}\text { Single crystal; } \\
\text { dislocation } \\
\text { density } \sim 6.6 \\
\times 10^{5} / \mathrm{cm}^{2}\end{array}$ & $\begin{array}{l}\text { Heterogeneous } \\
\text { isotopic exchange } \\
\text { between a } \\
\text { solid sample } \\
\text { and a gas. }\end{array}$ & $D^{*}$ & $850-1525$ & $1.2 \times 10^{-5}$ & 29,300 & $\begin{array}{l}\text { Possible extrinsic } \\
\text { diffusion; diffu- } \\
\text { sion rate was } \\
\text { found to depend } \\
\text { on dislocation } \\
\text { density. }\end{array}$ & [22] \\
\hline $\mathrm{SrTiO}_{3} \ldots \ldots$ & Sr............. & Not stated. & $\begin{array}{l}\mathrm{SrCO}_{3} \text { was reacted } \\
\text { with anatase to } \\
\text { form } \mathrm{SrTiO}_{3} ; \\
\text { compressed and } \\
\text { sintered; } 95 \% \text { the- } \\
\text { oretical density. }\end{array}$ & $\begin{array}{l}\text { Method of Kryukov } \\
\text { and Zhukhovitskii. }\end{array}$ & $D^{*}$ & $1000-3000$ & 4.0 & 70,000 & & [23] \\
\hline
\end{tabular}

a Impurity concentrations are given in ppm unless otherwise stated.

[1] F. C. Wagner, E. J. Bucur and M. A. Steinberg, Trans. ASM 48, 742 (1956).

[2] R. J. Wasilewski and G. L. Kehl, J. Inst. Metals 83, 94 (1954).

[3] A. V. Revyakin, Izv. Akad. Nauk SSSR, Otdel, Tekh. Nauk, Met. I Toplivo 113 (1961).

[4] P. Kofstad, P. B. Anderson, and O. J. Krudtaa, J. Less-Common Metals 3, 89 (1961).

[5] W. P. Roe, H. R. Palmer, and W. R. Opie, Trans. ASM 52, 191 (1960).

[6] F. Claisse and H. P. Koenig, Acta Met. 4, 650 (1956).

[7] G. V. Samsonov and A. P. Epik, Dopovidi Akad. Nauk Ukr. RSR [1], 67 (1964).

[8] A. A. Zhukhovitskii and V. A. Geodakyan, Dokl. Akad. Nauk SSSR 102, 301 (1955).

[9] M. L. Baskin, V. I. Tret'yakov, and I. N. Chaporova, Fiz. Metal. I Metalloved. 12, 860 (1961).

[10] A. A. Zhukhovitskii and S. N. Kryukov, Dokl. Akad. Nauk SSSR 90, 379 (1953).

[11] M. L. Baskin, V. I. Tret'yakov, and I. N. Chaporova, Fiz. Metal I Metalloved. 14, 422 (1962).

[12] J. Boltzmann, Ann. Physik 53, 959 (1894).

[13] C. Matano, Japan. J. Phys. 8, 109 (1933)

[14] T. Nakayama and T. Sasaki, Bull. Chem. Soc. Japan 36, 569 (1963).

[15] V. I. Izvekov and K. M. Gorbunova, Fiz. Metal. I Metalloved. 7, 713 (1959).

[16] O. W. Johnson, Phys. Rev. 136, A284 (1964).

[17] R. Haul, D. Just, and G. Dumbgen, Reactivity of Solids, p. 65 (Elsevier Publishing Co., Amsterdam, Netherlands, 1961).

[18] R. Haul and G. Dumbgen, J. Phys. Chem. Solids 26, 1 (1965).

[19] R. Lindner and Hj. Matzke, Z. Naturforsch. 15a, 1082 (1960).

[20] W. R. Sinclair and T. C. Loomis, Kinetics of High Temperature Processes, p. 58 (Technology Press, Cambridge, Mass., 1959).

[21] A. G. Verduch and R. Lindner, Arkiv. Kemi 5, 313 (1953).

[22] A. E. Paladino, L. G. Rubin, and J. S. Waugh, J. Phys. Chem. Solids 26, 391 (1965)

[23] P. Turlier, P. Bussiere, and M. Preffe, Compt. Rend. 250, 1649 (1960).

[24] R. I. Jaffe and I. E. Campbell, Trans. AIME 185, 646 (1949).

Zirconium

\begin{tabular}{|c|c|c|c|c|c|c|c|c|c|c|}
\hline $\begin{array}{l}\text { Solvent } \\
\text { medium }\end{array}$ & $\begin{array}{l}\text { Diffusing } \\
\text { element }\end{array}$ & Purity ${ }^{a}$ & $\begin{array}{l}\text { Preparation and } \\
\text { properties }\end{array}$ & Method & $\begin{array}{l}\text { Diffusion } \\
\text { coefficient }\end{array}$ & $\begin{array}{c}\text { Temperature } \\
\text { range, }{ }^{\circ} \mathrm{C}\end{array}$ & $D_{0} \mathrm{~cm}^{2} / \mathrm{s}$ & $Q \mathrm{cal} / \mathrm{mol}$ & Comments & Ref. \\
\hline$\beta-\mathrm{Zr} \ldots$ & C.... & $\begin{array}{l}\mathrm{Hf}, 700 ; \mathrm{N}, 140 ; \\
\quad \mathrm{Fe}, 400 ; \mathrm{Si}, 500 .\end{array}$ & $\begin{array}{l}\text { Zr-bars were } \\
\text { melted in an elec- } \\
\text { tron arc furnace; } \\
{ }^{14} \mathrm{C} \text { was added to } \\
\text { some specimens } \\
\text { which were } \\
\text { continually re- } \\
\text { melted to obtain } \\
\text { uniform distribu- } \\
\text { tion. }\end{array}$ & $\begin{array}{l}\text { Determination of } \\
\text { the concentration } \\
\text { gradient by radio- } \\
\text { isotope } \\
\text { distribution. }\end{array}$ & $\begin{array}{r}\tilde{D} \text { (chemical } \\
\text { diffusion) }\end{array}$ & $900-1260$ & 0.0048 & 26,700 & $\begin{array}{l}\tilde{D} \text { was assumed to } \\
\text { be independent of } \\
\text { concentration. }\end{array}$ & [1] \\
\hline$\beta-\mathrm{Zr} .$. & N.. & $\begin{array}{l}\mathrm{Hf}, 150 ; \mathrm{Fe}, 250 ; \\
\mathrm{Si}, 100 ; \mathrm{Sn}, 100 ; \\
\text { all others < } 100 \text {. }\end{array}$ & $\begin{array}{l}\mathrm{Zr} \text { : iodide-crystal } \\
\text { bars were pro- } \\
\text { duced by deBoer } \\
\text { process, double } \\
\text { arc melted, cold } \\
\text { rolled into rods. } \\
\text { Prepurified tank } \\
\mathrm{N}_{2} \text { was passed } \\
\text { over heated } \mathrm{Zr} \\
\text { turnings and } \\
\text { then through a } \\
\text { cold-trap. }\end{array}$ & $\begin{array}{l}\text { Determination of } \\
\text { the concentration } \\
\text { gradient by chemi- } \\
\text { cal analysis } \\
\text { (Kjeldahl). }\end{array}$ & $\widetilde{D}$ & $920-1640$ & 0.015 & 30,700 & $\begin{array}{l}\widetilde{D} \text { was assumed to } \\
\text { be independent of } \\
\text { concentration. }\end{array}$ & [2] \\
\hline
\end{tabular}

${ }^{\text {a }}$ Impurity concentrations are given in ppm unless otherwise stated. 
Zirconium-Continued

\begin{tabular}{|c|c|c|c|c|c|c|c|c|c|c|}
\hline $\begin{array}{l}\text { Solvent } \\
\text { medium }\end{array}$ & $\begin{array}{l}\text { Diffusing } \\
\text { element }\end{array}$ & Purity ${ }^{a}$ & $\begin{array}{l}\text { Preparation and } \\
\text { properties }\end{array}$ & Method & $\begin{array}{l}\text { Diffusion } \\
\text { coefficient }\end{array}$ & $\begin{array}{c}\text { Temperature } \\
\text { range, },{ }^{\circ} \mathrm{C}\end{array}$ & $D_{0} \mathrm{~cm}^{2} / \mathrm{s}$ & $Q \mathrm{cal} / \mathrm{mol}$ & Comments & Ref. \\
\hline$\beta-\mathrm{Zr} \ldots$ & N........... & $\begin{array}{l}\text { Hf, } 1.8-2.2 \text { w/o; } \\
\text { O, N, H, Fe, Si, } \\
\text { totaling }<100 .\end{array}$ & $\begin{array}{l}\text { Cylindrical speci- } \\
\text { mens were } \\
\text { machined from } \\
\text { outgassed iodide } \\
\mathrm{Zr} \text { bars. Com- } \\
\text { mercial } \mathrm{N}_{2} \text { was } \\
\text { dried by } \mathrm{Mg} \\
\text { perchlorate. }\end{array}$ & $\begin{array}{l}\text { Determination of } \\
\text { the concentration } \\
\text { gradient by chem- } \\
\text { ical analysis } \\
\text { (Kjeldahl). }\end{array}$ & $\tilde{D}$ & $900-1600$ & 0.003 & 33,600 & $\begin{array}{c}\widetilde{D} \text { was assumed to } \\
\text { be independent } \\
\text { of concentration. }\end{array}$ & [3] \\
\hline$\alpha-\operatorname{Zr} \ldots \ldots$ & $0 \ldots$ & $\begin{array}{l}\mathrm{O}, 300 ; \mathrm{C}, 150 \\
\mathrm{Fe}, 120 ; \mathrm{Al}, 50 \\
\mathrm{P}<75 ; \mathrm{Cr}, 40\end{array}$ & $\begin{array}{l}\text { Oxide films grown } \\
\text { on } \mathrm{Zr} \text { plates in } \\
\text { the presence of } \\
\mathrm{O}_{2} \text { at one atm } \\
\text { pressure. }\end{array}$ & $\begin{array}{l}\text { Determination of } \\
\text { the concentration } \\
\text { gradient by micro- } \\
\text { hardness } \\
\text { measurement and } \\
\text { analysis of the } \\
\text { reaction rates. }\end{array}$ & $\begin{array}{l}D_{\mathrm{kr}} \text { (grain. } \\
\text { boundary). } \\
D\end{array}$ & $\begin{array}{l}400-650 \\
650-850\end{array}$ & $\begin{array}{l}4.57 \times 10^{-4} \\
224\end{array}$ & $\begin{array}{l}35,000 \\
59,700\end{array}$ & $\begin{array}{l}\text { Electron micro- } \\
\text { scope studies of } \\
\text { fracture surfaces } \\
\text { of the metal sug- } \\
\text { gested that grain } \\
\text { boundary diffusion } \\
\text { was the predom- } \\
\text { inant mode at } \\
\text { low temperatures. }\end{array}$ & [4] \\
\hline$\alpha-\mathrm{Zr} \ldots \ldots$ & O.......... & $\begin{array}{l}\text { High temperature } \\
\text { samples: O, 1300; } \\
\text { N, } 240 ; \mathrm{Hf}, 2.04 \\
\text { w/o; Fe, 190; } \mathrm{Al}, \\
95 ; \mathrm{Ba}<650 ; \mathrm{Si} \\
\text { < } 650 . \text { Low tem- } \\
\text { perature sam- } \\
\text { ples: O, 600; Fe, } \\
\text { 170; Al, 290; Cr } \\
<650 .\end{array}$ & $\begin{array}{l}\text { Vacuum annealed } \\
\mathrm{Zr} \text { plates were ox- } \\
\text { idized in } \mathrm{O}_{2} \text { at } \\
\text { high temperatures } \\
\text { plus one sample } \\
\text { in } \mathrm{O}_{2} \text {-bearing } \mathrm{Na} \text {. } \\
\text { Low tempera- } \\
\text { ture oxidations } \\
\text { in } \mathrm{O}_{2} \text {-bearing } \mathrm{Na} \\
\text { or } \mathrm{CO}_{2} \text {. }\end{array}$ & $\begin{array}{l}\text { Determination of } \\
\text { the concentration } \\
\text { gradient by micro- } \\
\text { hardness meas- } \\
\text { urements. }\end{array}$ & $\begin{array}{l}\bar{D} \\
\tilde{D}\end{array}$ & $\begin{array}{l}400-700 \\
700-850\end{array}$ & $\begin{array}{l}9.13 \times 10^{-5} \\
69.2\end{array}$ & $\begin{array}{l}29,800 \\
56,190\end{array}$ & $\begin{array}{l}\text { Use of } \mathrm{O}_{2} \text {-bearing } \\
\text { sodium gave dif- } \\
\text { fusion results } \\
\text { similar to } \mathrm{O}_{2} \\
\text { oxidations. } D \\
\text { values were cal- } \\
\text { culated only over } \\
\text { range where lin- } \\
\text { earity existed be- } \\
\text { tween hardness } \\
\text { and } \mathrm{O} \text { concen- } \\
\text { tration. }\end{array}$ & [5] \\
\hline$\alpha-\mathrm{Zr} \ldots$ & O..... & $\begin{array}{l}\text { Low Hf content, } \\
\text { concentrations of } \\
\text { impurities not } \\
\text { stated. }\end{array}$ & $\begin{array}{l}\text { Rolled stock } \\
\text { annealed, pol- } \\
\text { ished and } \\
\text { etched; samples } \\
\text { anodized in KOH } \\
\text { solution to form } \\
\text { the oxide film. }\end{array}$ & $\begin{array}{l}\text { Time rate of } \\
\text { change of the } \\
\text { interference } \\
\text { color (i.e., } \\
\text { thickness) of } \\
\text { the oxide film } \\
\text { due to solution } \\
\text { of the film in } \\
\text { the metal. }\end{array}$ & $\tilde{D}$ & $400-585$ & 9.4 & 51,780 & $\begin{array}{c}\bar{D} \text { was assumed to } \\
\text { be independent } \\
\text { of concentration. }\end{array}$ & [6] \\
\hline$\alpha-\operatorname{Zr} \ldots \ldots$ & O......... & $\begin{array}{l}\mathrm{Al}, 45 ; \mathrm{C}, 50 ; \\
\mathrm{Fe}, 218 ; \mathrm{Hf}, 68 ; \\
\mathrm{Mo}, 25 ; \mathrm{O}, 140 ; \\
\mathrm{Si}<40 ; \mathrm{Zr}<50 \\
\text { all others }<25\end{array}$ & $\begin{array}{l}\text { Iodide-refined } \\
\mathrm{Zr} \text {, polished. } \mathrm{O}_{2} \\
\text { ( } 99.6 \mathrm{v} / \mathrm{o} \text { pure) } \\
\text { was passed } \\
\text { through heated } \\
\text { cupric oxide, } \\
\text { Ascarite, Linde } \\
5 \mathrm{~A} \text { molecular } \\
\text { sieves and cold } \\
\text { trap. }\end{array}$ & $\begin{array}{l}\text { Determination of } \\
\text { the concentration } \\
\text { gradient by micro- } \\
\text { hardness } \\
\text { measurements. }\end{array}$ & $\widetilde{D}$ & $400-850$ & 28.8 & 53,400 & $\begin{array}{c}\bar{D} \text { was assumed to } \\
\text { be independent } \\
\text { of concentration. }\end{array}$ & [7] \\
\hline$\alpha-\mathrm{Zr} \ldots \ldots$ & o......... & $\begin{array}{l}\text { Zr sample: } \mathrm{Fe} \\
\quad<100 ; \mathrm{Hf}, 55 ; \\
\text { C, } 80 ; \mathrm{O}, 160 ; \\
\text { all others }<50 . \\
\mathrm{Zr}-\mathrm{O} \text { alloy: } \mathrm{Fe}, \\
510 ; \mathrm{Al}, 200 ; \\
\mathrm{Hf},<300 ; \mathrm{C}, \\
200 ; \mathrm{H}, 57 ; \mathrm{N}, \\
89 ; \mathrm{O}, 3100 ; \text { all } \\
\text { others < <50. }\end{array}$ & $\begin{array}{l}\text { Oxygen enriched } \\
\mathrm{Zr} \text { disk was } \\
\text { pressure bonded } \\
\text { to low oxygen } \mathrm{Zr} \\
\text { disk at } 675^{\circ} \mathrm{C} \text { to } \\
\text { form the diffusion } \\
\text { couple. }\end{array}$ & $\begin{array}{l}\text { Determination of } \\
\text { the concentration } \\
\text { gradient by micro- } \\
\text { hardness } \\
\text { measurements. }\end{array}$ & $\widetilde{D}$ & $622-840$ & 3.3 & 49,500 & $\begin{array}{l}\text { Linearity of con- } \\
\text { centration to } \\
\text { hardness was } \\
\text { demonstrated for } \\
\text { O-Zr alloys with } \\
\text { less than } 2.4 \mathrm{a} / \mathrm{o} \\
\text { oxygen; linearity } \\
\text { of the probability } \\
\text { plot indicated } \\
\text { that } \widetilde{D} \text { was inde- } \\
\text { pendent of con- } \\
\text { centration. }\end{array}$ & {$[8]$} \\
\hline$\alpha-\mathrm{Zr} .$. & O....... & $\begin{array}{l}\mathrm{Al}, 100 ; \mathrm{Fe}, \\
1000 ; \mathrm{Si}, 425 ; \\
\mathrm{Ca}, 200 ; \mathrm{Hf}, \\
200 ; \mathrm{N}, 437 \\
\mathrm{O}, 800 ; \text { all } \\
\text { others }<50 .\end{array}$ & $\begin{array}{c}\mathrm{Zr} \text { bars; annealed, } \\
\text { polished and } \\
\text { etched; surface } \\
\text { oxidized in } \mathrm{O}_{2} .\end{array}$ & $\begin{array}{l}\text { Determination of } \\
\text { the concentration } \\
\text { gradient by micro- } \\
\text { hardness measure- } \\
\text { ments; gravimetric } \\
\text { determinations } \\
\text { also were used. }\end{array}$ & $\widetilde{D}$ & $\begin{array}{l}550-650 \\
650-850\end{array}$ & $\begin{array}{l}7.9 \times 10^{-5} \\
0.22\end{array}$ & $\begin{array}{l}30,500 \\
47,000\end{array}$ & $\begin{array}{c}\widetilde{D} \text { was assumed to } \\
\text { be independent of } \\
\text { concentration. } \\
\text { Grain boundary } \\
\text { diffusion probably } \\
\text { was important at } \\
\text { low temperatures. }\end{array}$ & [9] \\
\hline$\alpha-\mathrm{Zr}$. & $0 \ldots$ & & & & $\tilde{D}$ & $400-850$ & $\begin{aligned} & 6.81 \\
\pm & 2.9\end{aligned}$ & $\begin{array}{l}51,200 \\
\pm 800\end{array}$ & $\begin{array}{l}\text { Combined data; } \\
\text { data from only } \\
\text { the high tem- } \\
\text { perature ranges } \\
\text { of [5] and [9] } \\
\text { were used. This } \\
\text { agreement is } \\
\text { remarkable. }\end{array}$ & [5-9] \\
\hline $\mathrm{ZrB}_{2} \ldots$ & B.... & Not stated. & $\begin{array}{l}\text { Boride layer was } \\
\text { formed on a } \mathrm{Zr} \\
\text { cylinder by boron- } \\
\text { izing in boron } \\
\text { carbide and borax. }\end{array}$ & $\begin{array}{l}\text { Determination of } \\
\text { the concentration } \\
\text { gradient by micro- } \\
\text { hardness measure- } \\
\text { ments. }\end{array}$ & $\tilde{D}$ & $1100-1400$ & $1.26 \times 10^{-4}$ & 34,500 & \begin{tabular}{|}
$\bar{D}$ was assumed to \\
be independent of \\
concentration; \\
narrow homogeneity \\
range [11] may be a \\
major source of \\
error in the results.
\end{tabular} \mid & [10] \\
\hline
\end{tabular}

${ }^{a}$ Impurity concentrations are given in ppm unless otherwise stated. 
Zirconium-Continued

\begin{tabular}{|c|c|c|c|c|c|c|c|c|c|c|}
\hline $\begin{array}{l}\text { Solvent } \\
\text { medium }\end{array}$ & $\begin{array}{c}\text { Diffusing } \\
\text { element }\end{array}$ & Purity ${ }^{a}$ & $\begin{array}{l}\text { Preparation and } \\
\text { properties }\end{array}$ & Method & $\begin{array}{l}\text { Diffusion } \\
\text { coefficient }\end{array}$ & $\begin{array}{c}\text { Temperature } \\
\text { range, }{ }^{\circ} \mathrm{C}\end{array}$ & $D_{0} \mathrm{~cm}^{2} / \mathrm{s}$ & $Q \mathrm{cal} / \mathrm{mol}$ & Comments & Ref. \\
\hline $\mathrm{ZrC} \ldots \ldots$ & C... & Not stated. & $\begin{array}{l}\text { Carbide layer was } \\
\text { formed on a } \mathrm{Zr} \\
\text { cylinder by car- } \\
\text { burizing in } \\
\text { graphite. }\end{array}$ & $\begin{array}{l}\text { Determination of } \\
\text { the concentration } \\
\text { gradient by micro- } \\
\text { hardness }\end{array}$ & $\tilde{D}$ & $900-1300$ & 0.0344 & 41,000 & $\begin{array}{l}\tilde{D} \text { was assumed to } \\
\text { be independent of } \\
\text { concentration. }\end{array}$ & [10] \\
\hline $\mathrm{ZrC} \ldots \ldots$ & C.......... & $\begin{array}{c}\text { Zirconium: Cr, 84; } \\
\text { Fe, 570; Hf, 68; } \\
\text { O, 375; all oth- } \\
\text { ers <50. Graphite: } \\
\text { Ca, 140; Fe, 73; } \\
\text { all others < 50. }\end{array}$ & $\begin{array}{l}\mathrm{ZrC} \text { layer formed } \\
\text { between liquid } \mathrm{Zr} \\
\text { and graphite } \\
\text { crucible. }\end{array}$ & $\begin{array}{l}\text { Determination of } \\
\text { the rate of } \\
\text { growth of } \mathrm{ZrC} \\
\text { layer from pho- } \\
\text { tomicrographs of } \\
\text { longitudinal sec- } \\
\text { tions of the } \\
\text { samples. }\end{array}$ & $\tilde{D}$ & $2000-2860$ & 0.95 & 78,700 & $\begin{array}{l}D \text { was assumed to } \\
\text { be independent of } \\
\text { concentration. } \\
\text { The boundary } \\
\text { concentrations } \\
\text { were taken from } \\
\text { phase diagrams } \\
{[13,14] .}\end{array}$ & [12] \\
\hline $\mathrm{ZrO}_{2} \ldots$ & $0 \ldots$ & $\begin{array}{l}\text { Zr substrate: } \mathrm{O} \\
\quad 300 ; \mathrm{C}, 150 ; \mathrm{Fe} \\
\text { 120; } \mathrm{Al}, 50 ; \mathrm{P} \\
\quad \text { < } 75 ; \mathrm{Cr}, 40 .\end{array}$ & $\begin{array}{l}\text { Oxide layers } \\
\text { grown on } \mathrm{Zr} \\
\text { plates in } \mathrm{O}_{2} ; \\
\text { monoclinic oxide } \\
\text { studied. }\end{array}$ & $\begin{array}{l}\text { Determination of } \\
\text { rate of oxide } \\
\text { layer growth }\end{array}$ & $\tilde{D}$ & $400-850$ & $1.05 \times 10^{-3}$ & 29,300 & & [4] \\
\hline $\mathrm{ZrO}_{2} \ldots$ & $\begin{array}{l}\text { O............. } \\
\text { vacancies }\end{array}$ & $\begin{array}{l}\text { Impurities in } \\
\text { metal substrate } \\
\text { not stated. }\end{array}$ & $\begin{array}{l}\text { Oxide layers } \\
\text { formed on crystal } \\
\text { bar } \mathrm{Zr} \text { in } \mathrm{O}_{2} \text {, } \\
\text { then the anion } \\
\text { vacancy concen- } \\
\text { tration in the } \\
\text { oxide equilibrated } \\
\text { by annealing in } \\
\text { vacuum; approxi- } \\
\text { mate composition, } \\
\mathrm{ZrO}_{1.926} \text {; mono- } \\
\text { clinic phase. }\end{array}$ & $\begin{array}{l}\text { Interruption } \\
\text { kinetics tech- } \\
\text { nique of Rosen- } \\
\text { burg [16]: rate } \\
\text { of oxygen up- } \\
\text { take by oxygen } \\
\text { deficient } \mathrm{ZrO}_{2-x} \text {. }\end{array}$ & $\tilde{D}$ & $300-386$ & $9.0 \times 10^{-4}$ & 28,700 & $\begin{array}{l}\text { Comparison of } \tilde{D} \text { for } \\
\text { vacancy diffusion } \\
\text { with O-anion dif- } \\
\text { fusion requires } \\
\text { the use of an un- } \\
\text { known correlatior } \\
\text { factor. The anion } \\
\text { vacancy concen- } \\
\text { tration in the } \\
\text { deficient oxide } \\
\text { was } \sim 7 \times 10^{-3} \mathrm{~g} \\
\text { atom } / \mathrm{cm}^{3} \\
\left(386^{\circ} \mathrm{C}\right) \text {. }\end{array}$ & [15] \\
\hline $\mathrm{ZrO}_{1.994} \ldots$ & $0 \ldots \ldots$ & $\begin{array}{l}\mathrm{SiO}_{2}, 0.15 \mathrm{w} / \mathrm{o} ; \\
\mathrm{TiO}_{2}, 50 ; \mathrm{Fe}_{2} \mathrm{O}_{3}, \\
\text { 30; } \mathrm{MgO}, 300 .\end{array}$ & $\begin{array}{l}\text { Hot pressed non- } \\
\text { stoichiometric } \\
\text { oxide compacts; } \\
\text { density } 5.82 \\
\text { g/ } / \mathrm{cm}^{3} ; \text { mono- } \\
\text { clinic phase. }\end{array}$ & $\begin{array}{l}\text { Determination of } \\
\text { the rate of migra- } \\
\text { tion of stoichio- } \\
\text { metric oxide front } \\
\text { into nonstoichio- } \\
\text { metric grains. }\end{array}$ & $\tilde{D}$ & $700-1000$ & 0.055 & 33,400 & $\begin{array}{l}\text { Rapid grain bound- } \\
\text { ary diffusion into } \\
\text { the compacts was } \\
\text { noted so that the } \\
\text { re-oxidation of in- } \\
\text { dividual grains was } \\
\text { measured. A com- } \\
\text { parison with [4] } \\
\text { and [15] above } \\
\text { suggests that (a) } \\
\text { grain boundary ef- } \\
\text { fects may be } \\
\text { small in coherent } \\
\text { oxide layers, (b) } \\
\widetilde{D} \text { does not vary } \\
\text { strongly with com- } \\
\text { position nor with } \\
\text { crystallographic } \\
\text { direction, and (c) } \\
\text { the correlation } \\
\text { factor may be } \\
\text { near unity. }\end{array}$ & [17] \\
\hline $\begin{array}{l}\mathrm{Zr}_{0.84^{-}} \\
\quad \mathrm{Ca}_{0.16^{-}} \\
\quad \mathrm{O}_{1.84}\end{array}$ & Сa....... & $\begin{array}{l}99.95 \mathrm{w} / \mathrm{o} \mathrm{ZrO}_{2} \\
\text { and } \mathrm{CaCO}_{3} \text { used } \\
\text { in fabrication; } \\
\mathrm{W}<100 \text {. }\end{array}$ & $\begin{array}{l}\text { Calcined mixture } \\
\text { of } \mathrm{ZrO}_{2} \text { and } \\
\mathrm{CaCO}_{3} \text { pressed } \\
\text { into pellets, fused } \\
\text { with plasma } \\
\text { torch; grain size } \\
0.2-1.0 \mathrm{~mm} ; \text { po- } \\
\text { rosity } 0.0-0.5 \% \\
\text { cubic phase. }\end{array}$ & $\begin{array}{l}\text { Determination of } \\
\text { the concentration } \\
\text { gradient by radio- } \\
\text { isotope distribu- } \\
\text { tions. }\end{array}$ & $D^{*}$ & $1700-2100$ & 0.444 & 100,200 & $\begin{array}{l}\text { The error due to } \\
\text { grain boundary } \\
\text { diffusion was } \\
\text { within the experi- } \\
\text { mental uncertainty } \\
\text { of the data. }\end{array}$ & [18] \\
\hline $\begin{array}{l}\mathrm{Zr}_{0.92^{-}} \\
\quad \mathrm{Ca}_{0.08^{-}} \\
\quad \mathrm{O}_{1.85}\end{array}$ & O... & $\begin{array}{c}\mathrm{HfO}_{2}, 1.5 \% ; \mathrm{SiO}_{2}, \\
0.5 \% ; \mathrm{MgO}, 0.20 \% \\
\mathrm{Fe}_{2} \mathrm{O}_{3}, 0.10 \% \\
\mathrm{Al}_{2} \mathrm{O}_{3}, 0.17 \% \\
\mathrm{TiO}_{2}, 0.11 \% \\
\text { (weight percent). }\end{array}$ & $\begin{array}{l}\text { Tube closed at one } \\
\text { end; density } 5.4 \\
\text { g/cm³ } \\
\sim 5 \% \text { porosity } \\
\text { connected; cubic } \\
\text { phase. }\end{array}$ & $\begin{array}{l}\text { Determination of } \\
\text { the rate of per- } \\
\text { meation of } \mathrm{O}_{2} \\
\text { through the walls. }\end{array}$ & $\tilde{D}$ & $1100-1600$ & 185 & 57,600 & $\begin{array}{c}\text { A large drop in } \mathrm{O}_{2} \\
\text { partial pressure } \\
\text { between the inside } \\
\text { and outside caused } \\
\text { a large chemical } \\
\text { potential gradient. }\end{array}$ & [19] \\
\hline $\begin{array}{l}\mathrm{Zr}_{0.858^{-}} \\
\mathrm{Ca}_{0.142^{-}} \\
\mathrm{O}_{1.858}\end{array}$ & O..... & Not stated. & $\begin{array}{l}\text { Polycrystalline } \\
\text { samples: porosity } \\
3-6 \% \text {; grain size } \\
100 \mu \mathrm{m} \text {. Single } \\
\text { crystal sample: } \\
\text { verneuil method; } \\
\text { crack-free speci- } \\
\text { mens used; cubic } \\
\text { phase. }\end{array}$ & $\begin{array}{l}\text { Heterogeneous } \\
\text { isotopic exchange } \\
\text { between solid } \\
\text { sample and }{ }^{18} \mathrm{O} \text { - } \\
\text { enriched } \mathrm{O}_{2} \text {. }\end{array}$ & $D^{*}$ & $780-1100$ & 0.018 & 31,200 & $\begin{array}{l}D_{0} \text { and } Q \text { were } \\
\text { determined from } \\
\text { the combined data } \\
\text { from both types } \\
\text { of samples. }\end{array}$ & [20] \\
\hline $\begin{array}{l}\mathrm{Zr}_{0.85^{-}} \\
\quad \mathrm{Ca}_{0.15^{-}} \\
\mathrm{O}_{1.85}\end{array}$ & $0 \ldots$ & Not stated. & $\begin{array}{l}\text { Arc-fused, } \\
\text { polycrystalline } \\
\text { spheres. }\end{array}$ & $\begin{array}{l}\text { Heterogenous } \\
\text { isotopic exchange. }\end{array}$ & $D^{*}$ & $700-1100$ & $6.9 \times 10^{-3}$ & 30,400 & $\mid \begin{array}{l}D_{0} \text { and } Q \text { are given } \\
\text { as recalculated by } \\
\text { Simpson and } \\
\text { Carter }\lceil 20\rceil .\end{array}$ & [21] \\
\hline
\end{tabular}

${ }^{a}$ Impurity concentrations are given in ppm unless otherwise stated. 
Zirconium-Continued

\begin{tabular}{|c|c|c|c|c|c|c|c|c|c|c|}
\hline $\begin{array}{l}\text { Solvent } \\
\text { medium }\end{array}$ & $\begin{array}{l}\text { Diffusing } \\
\text { element }\end{array}$ & Purity a & $\begin{array}{l}\text { Preparation and } \\
\text { properties }\end{array}$ & Method & $\begin{array}{l}\text { Diffusion } \\
\text { coefficient }\end{array}$ & $\begin{array}{c}\text { Temperature } \\
\text { range, }{ }^{\circ} \mathrm{C}\end{array}$ & $D_{0} \mathrm{~cm}^{2} / \mathrm{s}$ & $Q \mathrm{cal} / \mathrm{mol}$ & Comments & Ref. \\
\hline $\begin{array}{c}\mathrm{Zr}_{0.84^{-}} \\
\mathrm{Ca}_{0.16^{-}} \\
\mathrm{O}_{1.84} \\
\mathrm{Zr}_{0.88^{-}} \\
\mathrm{Ca}_{0.12^{-}} \\
\mathrm{O}_{1.88}\end{array}$ & Zr.......... & $\begin{array}{l}99.95 \mathrm{w} / \mathrm{o} \mathrm{ZrO}_{2} \\
\text { and } \mathrm{CaCO}_{3} \text { used } \\
\text { in fabrication; } \\
\mathrm{W}<100 \text {. }\end{array}$ & $\begin{array}{l}\text { Calcined mixture } \\
\text { of } \mathrm{ZrO}_{2} \text { and } \mathrm{CaCO}_{3} \\
\text { pressed into pel- } \\
\text { lets, fused with } \\
\text { plasma torch; grain } \\
\text { size } 0.2-1.0 \mathrm{~mm} ; \\
\text { porosity } 0.0-0.5 \% \text {; } \\
\text { cubic phase. }\end{array}$ & $\begin{array}{l}\text { Determination of } \\
\text { the concentration } \\
\text { gradient by radio- } \\
\text { isotope distri- } \\
\text { bution. }\end{array}$ & $D^{*}$ & $1700-2150$ & 0.035 & 92,500 & $\begin{array}{l}D_{0} \text { and } Q \text { represent } \\
\text { the combined results } \\
\text { for the two composi- } \\
\text { tions. The error } \\
\text { due to grain boundary } \\
\text { diffusion was within } \\
\text { the experimental } \\
\text { error of the data. }\end{array}$ & [18] \\
\hline
\end{tabular}

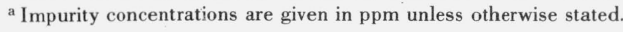

[1] L. V. Pavlinov and V. N. Bykov, Fiz. Metal. I Metalloved. 19, 397 (1965).

[2] M. W. Mallett, J. Belle, and B. B. Cleland, J. Electrochem. Soc. 101, 1 (1954); U.S. At. Energy Comm. Report BMI-829 (1953).

[3] M. W. Mallett, E. M. Baroody, H. R. Nelson, and C. A. Papp, J. Electrochem. Soc. 100, 103 (1953).

[4] J. Debuigne and P. Lehr, Compt. Rend. 256, 1113 (1963); Conf. Corrosion of Reactor Materials 2, p. 105 (International Atomic Energy Agency, Vienna, 1962).

[5] M. Davis, K. R. Montgomery, and J. Standring, J. Inst. Metals 89, 172 (1961).

[6] J. P. Pemsler, J. Electrochem. Soc. 105, 315 (1958).

[7] R. J. Hussey and W. W. Smeltzer, J. Electrochem. Soc. 111, 564 (1964); ibid. 111, 1221 (1964).

[8] J. J. Kearns and J. N. Chirigos, U.S. At. Energy Comm. Report WAPD-TM-306 (1962).

[9] G. Beranger and P. Lacombe, J. Nucl. Mater. 16, 190 (1965).

[10] G. V. Samsonov and A. P. Epik, Dopovidi Akad. Nauk Ukr. RSR [1] 67 (1964)

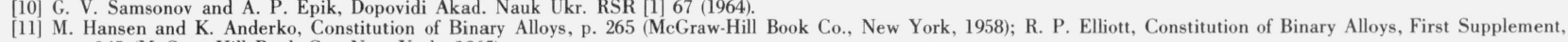
p. 145 (McGraw-Hill Book Co., New York, 1965).
L. M. Adelsberg, L. H. Cadoff, and J. M. Tobin, Trans. AIME 236, 972 (1966).

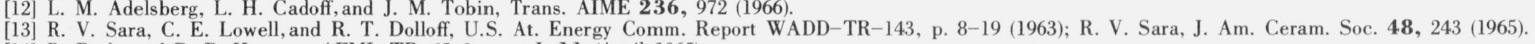

[14] R. Rudy and D. P. Harmon, AFML-TR-65-2, part I, 11 (April 1965).

[15] T. Smith, J. Electrochem. Soc. 112, 560 (1965).

[16] A. J. Rosenburg, J. Electrochem. Soc. 107, 795 (1960).

[17] D. L. Douglass, Conf. Corrosion of Reactor Materials, 2, p. 224 (International Atomic Energy Agency, Vienna, 1962).

[18] W. H. Rhodes and R. E. Carter, J. Am. Ceram. Soc. 49, 244 (1966).

[19] A. W. Smith, F. W. Meszaros, and C. D. Amata, J. Am. Ceram. Soc. 49, 240 (1966)

[20] L. A. Simpson and R. E. Carter, J. Am. Ceram. Soc. 49, 139 (1966).

$[21]$ W. D. Kingery, J. Pappis, M. E. Doty, and D. C. Hill, J. Am. Ceram. Soc. 42, 393 (1959).

\section{References}

[1] J. Boltzmann, Ann. Physik. 53, 959 (1894).

[2] C. Matano, Japan. J. Phys. 8, 109 (1933).

[3] J. C. Fisher, J. Appl. Phys. 22, 74 (1951).
[4] R. T. P. Whipple, Phil. Mag. 45, 1225 (1954).

[5] H. S. Levine and C. J. MacCallum, J. Appl. Phys. 31, 595 (1960).

[6] T. Suzuoka, Trans. Japan. Inst. Metals 2, 25 (1961).

(Paper 72A2-492) 